\title{
Spectrum of a diffusion operator with coefficient changing sign over a small inclusion
}

\author{
Lucas Chesnel ${ }^{1}$, Xavier Claeys $^{2}$, Sergei A. Nazarov ${ }^{3,4,5}$ \\ 1 Centre de Mathématiques Appliquées, bureau 2029, École Polytechnique, 91128 Palaiseau Cedex, \\ France; \\ ${ }^{2}$ Laboratory Jacques Louis Lions, University Pierre et Marie Curie, 4 place Jussieu, 75005 Paris, \\ France; \\ ${ }^{3}$ Faculty of Mathematics and Mechanics, St. Petersburg State University, Universitetsky prospekt, \\ 28, 198504, Peterhof, St. Petersburg, Russia; \\ 4 Laboratory for mechanics of new nanomaterials, St. Petersburg State Polytechnical University, \\ Polytekhnicheskaya ul, 29, 195251, St. Petersburg, Russia; \\ ${ }^{5}$ Laboratory of mathematical methods in mechanics of materials, Institute of Problems of Mechanical \\ Engineering, Bolshoj prospekt, 61, 199178, V.O., St. Petersburg, Russia; \\ E-mail: chesnel@cmap.polytechnique.fr, claeys@ann.jussieu.fr, srgnazarov@yahoo.co.uk
}

(August 4, 2018)

\begin{abstract}
We study a spectral problem $\left(\mathscr{P}^{\delta}\right)$ for a diffusion like equation in a $3 \mathrm{D}$ domain $\Omega$. The main originality lies in the presence of a parameter $\sigma^{\delta}$, whose sign changes on $\Omega$, in the principal part of the operator we consider. More precisely, $\sigma^{\delta}$ is positive on $\Omega$ except in a small inclusion of size $\delta>0$. Because of the sign-change of $\sigma^{\delta}$, for all $\delta>0$ the spectrum of $\left(\mathscr{P}^{\delta}\right)$ consists of two sequences converging to $\pm \infty$. However, at the limit $\delta=0$, the small inclusion vanishes so that there should only remain positive spectrum for $\left(\mathscr{P}^{\delta}\right)$. What happens to the negative spectrum? In this paper, we prove that the positive spectrum of $\left(\mathscr{P}^{\delta}\right)$ tends to the spectrum of the problem without the small inclusion. On the other hand, we establish that each negative eigenvalue of $\left(\mathscr{P}^{\delta}\right)$ behaves like $\delta^{-2} \mu$ for some constant $\mu<0$. We also show that the eigenfunctions associated with the negative eigenvalues are localized around the small inclusion. We end the article providing 2D numerical experiments illustrating these results.
\end{abstract}

Key words. Negative materials, small inclusion, plasmonics, metamaterial, sign-changing coefficients, eigenvalues, asymptotics, singular perturbation.

\section{Introduction}

Let $\Omega$ and $\Xi_{-}$be three-dimensional domains, i.e. bounded and connected open subsets of $\mathbb{R}^{3}$, with boundaries $\partial \Omega$ and $\Gamma:=\partial \Xi_{-}$that admit $\mathscr{C}^{\infty}$ regularity. Assume that $\Omega, \Xi_{-}$contain the origin $O$ and that there holds $\Xi_{-} \subset \Omega$. For $\delta \in(0 ; 1]$, we introduce the sets (see Figure 1)

$$
\begin{array}{ll}
\Omega_{-}^{\delta}:=\delta \Xi_{-} \\
\Omega_{+}^{\delta}:=\quad \Omega \backslash \overline{\Omega_{-}^{\delta}} \\
\Gamma^{\delta}:=\partial \Omega_{-}^{\delta} .
\end{array}
$$

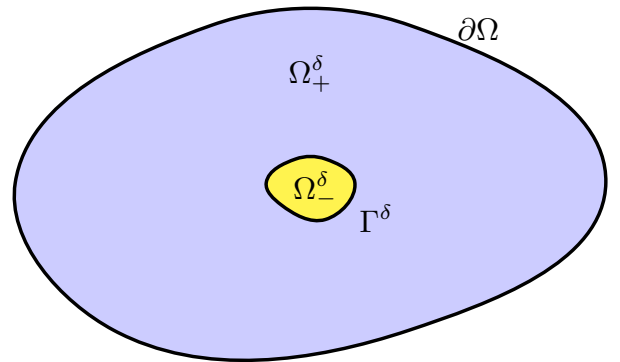

Figure 1: Schematic view of the geometry.

Define $\sigma^{\delta}: \Omega \rightarrow \mathbb{R}$ by $\sigma=\sigma_{-}$on $\overline{\Omega_{-}^{\delta}}$ and $\sigma=\sigma_{+}$on $\Omega_{+}^{\delta}$, where $\sigma_{-}<0$ and $\sigma_{+}>0$ are constants. Throughout the paper, if $u$ is a measurable function on $\Omega$, we shall denote $u_{ \pm}:=\left.u\right|_{\Omega_{ \pm}^{\delta}}$. For any open set $\omega \subset \mathbb{R}^{3}$, the space $\mathrm{L}^{2}(\omega)$ will refer to the set of square integrable functions defined on $\omega$, equipped 
with the scalar product $(u, v)_{\omega}=\int_{\omega} u \bar{v} d \boldsymbol{x}$ and the norm $\|u\|_{\omega}:=\sqrt{(u, u)_{\omega}}$. In the present article, we wish to study the following spectral problem involving a Dirichlet boundary condition:

$$
\mid \begin{aligned}
& \text { Find }\left(\lambda^{\delta}, u^{\delta}\right) \in \mathbb{C} \times\left(\mathrm{H}_{0}^{1}(\Omega) \backslash\{0\}\right) \text { such that } \\
& -\operatorname{div}\left(\sigma^{\delta} \nabla u^{\delta}\right)=\lambda^{\delta} u^{\delta} \quad \text { in } \Omega .
\end{aligned}
$$

In Poblem (1), $\lambda^{\delta}$ is the spectral parameter. Moreover, $\mathrm{H}_{0}^{1}(\Omega)$ stands for the subspace of functions of the Sobolev space $\mathrm{H}^{1}(\Omega)$ vanishing on $\partial \Omega$. It is endowed with the norm $\|u\|_{\mathrm{H}_{0}^{1}(\Omega)}:=\|\nabla u\|_{\Omega}$. We regard $-\operatorname{div}\left(\sigma^{\delta} \nabla \cdot\right)$ as the unbounded operator $\mathrm{A}^{\delta}: D\left(\mathrm{~A}^{\delta}\right) \rightarrow \mathrm{L}^{2}(\Omega)$ defined by

$$
\mid \begin{aligned}
& \mathrm{A}^{\delta} v=-\operatorname{div}\left(\sigma^{\delta} \nabla v\right) \\
& D\left(\mathrm{~A}^{\delta}\right):=\left\{v \in \mathrm{H}_{0}^{1}(\Omega) \mid \operatorname{div}\left(\sigma^{\delta} \nabla v\right) \in \mathrm{L}^{2}(\Omega)\right\} .
\end{aligned}
$$

Since the interface $\Gamma^{\delta}=\partial \Omega_{-}^{\delta}$ between the two subdomains is smooth, one can verify that when the contrast $\kappa_{\sigma}:=\sigma_{-} / \sigma_{+}$satisfies $\kappa_{\sigma} \neq-1, \mathrm{~A}^{\delta}$ fits the standard framework of [23, 44] for dealing with transmission problems. This leads to the following result (for a detailed discussion for this particular problem, see also [43]).

Proposition 1.1. Assume that $\kappa_{\sigma}=\sigma_{-} / \sigma_{+} \neq-1$. Then for all $\delta \in(0 ; 1]$, the operator $\mathrm{A}^{\delta}$ is densely defined, closed, self-adjoint and admits compact resolvent.

Therefore, for a fixed $\delta>0$, we can study the spectrum of $\mathrm{A}^{\delta}$. Because $\sigma^{\delta}$ changes sign on $\Omega$, this spectrum is not bounded from below nor from above. More precisely, we have the following results (see [43] and [7]).

Proposition 1.2. Assume that $\kappa_{\sigma} \neq-1$. Then for all $\delta \in(0 ; 1]$, the spectrum of $\mathrm{A}^{\delta}$ consists in two sequences, one nonnegative and one negative, of real eigenvalues of finite multiplicity:

$$
\cdots \lambda_{-n}^{\delta} \leq \cdots \leq \lambda_{-1}^{\delta}<0 \leq \lambda_{1}^{\delta} \leq \lambda_{2}^{\delta} \leq \cdots \leq \lambda_{n}^{\delta} \ldots .
$$

In the sequences above, the numbering is chosen so that each eigenvalue is repeated according to its multiplicity. Moreover, there holds $\lim _{n \rightarrow+\infty} \lambda_{ \pm n}^{\delta}= \pm \infty$.

In Proposition 1.1, the assumption $\kappa_{\sigma} \neq-1$ is important and in the sequel, we should not depart from it. The case $\kappa_{\sigma}=-1$ is rather pathological and is beyond the scope of the present article.

Proposition 1.2 indicates that for all $\delta \in(0 ; 1], \mathfrak{S}\left(\mathrm{A}^{\delta}\right)$ (the spectrum of $\mathrm{A}^{\delta}$ ) contains a sequence of eigenvalues which tends to $-\infty$. On the other hand, when $\delta$ goes to zero, the small inclusion vanishes so that the parameter $\sigma^{\delta}$ becomes strictly positive at the limit $\delta=0$. As a consequence, one could expect to obtain only positive spectrum for the operator $\mathrm{A}^{\delta}$ when $\delta \rightarrow 0$. The question we want to answer in this paper can be formulated as follows: what happens to the negative spectrum of $\mathrm{A}^{\delta}$ when the small inclusion shrinks?

Problems of small inclusions or small holes have a long history in asymptotic analysis. The case where $\Omega_{-}^{\delta}$ is a hole (with Dirichlet of Neumann condition on $\partial \Omega_{-}^{\delta}$ ) in $2 \mathrm{D} / 3 \mathrm{D}$ has been studied in detail in $[28,29]$ (see also the references therein). The configuration where $\Omega_{-}^{\delta}$ contains a positive material with a concentrated mass has been investigated in [45, 38, 30, 9] (see also the review [24]). In this context, the asymptotics of eigenpairs of elliptic operators has been considered in [27, 28, 26].

A remarkable feature of Problem (1) is the change of sign of the parameter appearing in the principal part of the operator $\mathrm{A}^{\delta}$. This is what makes it non-standard. In [35, 32, 34], the authors have examined the asymptotics of eigenpairs in situations where a sign changing coefficient arises in the compact part of the spectral problem under study. Yet, to our knowledge, asymptotics of the eigenpairs with a sign changing coming into play in the principal part of the operator has never been considered before.

The motivation for investigating Problem (1) comes from electromagnetics and in particular from 
the so-called surface plasmon polaritons as well as metamaterials that both offer a wide range of new technological perspectives. The surface plasmon polaritons are waves which propagate at the interface between a metal and a classical dielectric in the visible range. They appear because at optical frequencies, neglecting dissipation effects, the permittivity of a metal can be negative [39, $3,11,48,16]$. The metamaterials (see [1] for an overview) are artificial materials, made of small resonators periodically arranged so as to obtain macroscopic media with exotic permittivity $\varepsilon$ and/or permeability $\mu$. In this field, one of the goals consists in achieving negative $\varepsilon$ and/or $\mu$. We emphasize that in the present article, we consider a problem involving a material such that only one physical parameter $(\varepsilon$ or $\mu$ ) takes negative values. When additionally there is a sign changing function in the right hand side of Equation (1), complex spectrum can appear and the analysis we propose does not apply.

The outline of the paper is the following. In Section 2, we start by presenting the features of the two limit operators $\mathrm{A}^{0}$ and $\mathrm{B}^{\infty}$ (see their definition in (6) and (10)) which appear naturally in the study of the spectrum of $\mathrm{A}^{\delta}$ when $\delta$ tends to zero. In Section 3 , we give an asymptotic expansion of the solution of the source term problem associated with (1) as $\delta \rightarrow 0$. In order to justify this asymptotic expansion, we establish uniform boundedness of the inverse of $\mathrm{A}^{\delta}$ in terms of weighted norms involving the small parameter $\delta$. The proof of this important result is difficult because of the change of sign of the parameter $\sigma^{\delta}$. Our approach is based on the technique of overlapping cut-off functions introduced in [29, Chap 2], [31]. Then, we make use of this uniform boundedness result in order to show that $\left(\mathrm{A}^{\delta}\right)^{-1}$ converges strongly to $\left(\mathrm{A}^{0}\right)^{-1}$ in the operator norm as $\delta \rightarrow 0$. This allows to prove directly, in Section 4 , that the positive part of the spectrum of $\mathrm{A}^{\delta}$ converges to the spectrum of $\mathrm{A}^{0}$. This will also imply that the negative eigenvalues of $\mathrm{A}^{\delta}$ all diverge to $-\infty$. Section 5 then focuses on a sharper study of the negative part of the spectrum of $\mathrm{A}^{\delta}$. In particular, we show that all negative eigenvalues admit a behaviour of the form $\delta^{-2} \mu$, with $\mu<0$. In the last section, we illustrate these theoretical results with 2D numerical experiments. The two main results of the paper, respectively for the positive and negative spectrum of $\mathrm{A}^{\delta}$, are formulated in Theorem 4.1 and Theorem 5.1.

\section{Limit problems}

In the sequel, we will provide an asymptotic expansion of the eigenpairs of Problem (1) as $\delta$ tends to zero. This asymptotic expansion will involve the spectral parameters of some operators associated with two limit problems independent of $\delta$. The goal of the present section is to introduce these operators and to provide their main features. Before proceeding further, we introduce a set of cut-off functions which will be useful in our analysis. Let $\psi$ and $\chi$ be two elements of $\mathscr{C}^{\infty}(\mathbb{R},[0 ; 1])$ such that

$$
\psi(r)+\chi(r)=1, \quad \psi(r)=1 \text { for } r \leq 1, \quad \text { and } \quad \psi(r)=0 \text { for } r \geq 2 .
$$

For $t>0$, we shall denote $\psi_{t}$ and $\chi_{t}$ the functions (see Figure 2) such that

$$
\psi_{t}(r)=\psi(r / t) \quad \text { and } \quad \chi_{t}(r)=\chi(r / t) .
$$
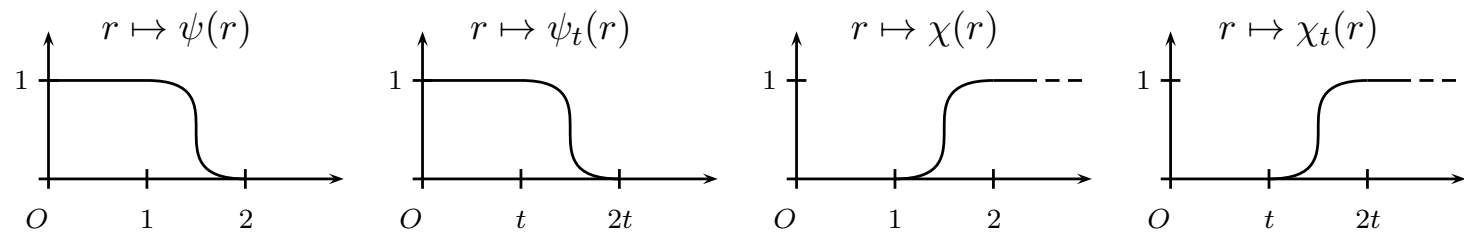

Figure 2: Cut-off functions.

In order to simplify the presentation and without restriction, we shall assume that the domains $\Omega$ and $\Xi_{-}$are such that $\overline{\mathrm{B}(O, 2)} \subset \Omega, \Xi_{-} \subset \overline{\mathrm{B}(O, 1)}$ so that the support of $\psi$ is included in $\Omega$ and so that $\psi=1$ on $\Xi_{-}$. Here and throughout the paper, $\mathrm{B}(O, d)$ denotes the open ball of $\mathbb{R}^{3}$, centered at $O$ and of radius $d>0$. If the domains $\Omega, \Xi_{-}$do not satisfy this assumption, we modify the cut-off functions accordingly. 


\section{$2.1 \quad$ Far field operator}

As $\delta$ tends to zero, the small inclusion of negative material disappears. In other words, there holds $\sigma^{\delta} \rightarrow \sigma_{+}$a.e. in $\Omega$. This leads us to introduce the far field operator $\mathrm{A}^{0}: D\left(\mathrm{~A}^{0}\right) \rightarrow \mathrm{L}^{2}(\Omega)$ such that

$$
\mid \begin{aligned}
& \mathrm{A}^{0} v=-\sigma_{+} \Delta v \\
& D\left(\mathrm{~A}^{0}\right):=\left\{v \in \mathrm{H}_{0}^{1}(\Omega) \mid \Delta v \in \mathrm{L}^{2}(\Omega)\right\} .
\end{aligned}
$$

Since $\partial \Omega$ is smooth, $D\left(\mathrm{~A}^{0}\right)$ coincides with $\mathrm{H}^{2}(\Omega) \cap \mathrm{H}_{0}^{1}(\Omega)$. Moreover, the operator $\mathrm{A}^{0}$ is bijective from $D\left(\mathrm{~A}^{0}\right)$ to $\mathrm{L}^{2}(\Omega)$ and its spectrum forms a discrete sequence of eigenvalues:

$$
\mathfrak{S}\left(\mathrm{A}^{0}\right)=\left\{\mu_{n}\right\}_{n \geq 1} \quad \text { with } \quad 0<\mu_{1}<\mu_{2} \leq \cdots \leq \mu_{n} \ldots \underset{n \rightarrow+\infty}{\rightarrow}+\infty .
$$

In (7), each eigenvalue is repeated according to its multiplicity. On the other hand, if $v_{n}$ is an eigenfunction associated with the eigenvalue $\mu_{n}$, then $v_{n} \in \mathscr{C}^{\infty}(\bar{\Omega})$ (at least when $\partial \Omega$ is of class $\mathscr{C}^{\infty}$ ).

In the sequel, we shall need sharp estimates for the behaviour of the eigenfunctions of $\mathrm{A}^{0}$ at $O$. Following [22], we shall express them in weighted norms. Let $\mathscr{C}_{0}^{\infty}(\bar{\Omega} \backslash\{O\})$ refer to the set of infinitely differentiable functions supported in $\bar{\Omega} \backslash\{O\}$. For $\beta \in \mathbb{R}$ and $k \geq 0$, we define the Kondratiev space $\mathrm{V}_{\beta}^{k}(\Omega)$ as the completion of $\mathscr{C}_{0}^{\infty}(\bar{\Omega} \backslash\{O\})$ in the norm

$$
\|v\|_{\mathrm{V}_{\beta}^{k}(\Omega)}:=\left(\sum_{|\alpha| \leq k} \int_{\Omega} r^{2(\beta+|\alpha|-k)}\left|\partial_{x}^{\alpha} v\right|^{2} d \boldsymbol{x}\right)^{1 / 2} .
$$

In (8), $r=|\boldsymbol{x}|$ denotes the distance to the origin $O$. We define the spaces $\mathrm{V}_{\beta}^{k}\left(\Omega_{ \pm}^{\delta}\right)$ like $\mathrm{V}_{\beta}^{k}(\Omega)$, replacing $\Omega$ by $\Omega_{ \pm}^{\delta}$ in (8). Of course, since $O \notin \Omega_{+}^{\delta}$, the space $\mathrm{V}_{\beta}^{k}\left(\Omega_{+}^{\delta}\right)$ coincides with $\mathrm{H}^{k}\left(\Omega_{+}^{\delta}\right)$ for all $\beta \in \mathbb{R}$. To deal with homogeneous Dirichlet boundary condition, we shall consider functions belonging to the space $\stackrel{\mathrm{V}}{\beta}_{\beta}^{1}(\Omega):=\left\{v \in \mathrm{V}_{\beta}^{1}(\Omega) \mid v=0\right.$ on $\left.\partial \Omega\right\}$. Then, we introduce the continuous operators $\mathcal{A}_{\beta}: D\left(\mathcal{A}_{\beta}\right) \rightarrow \mathrm{V}_{\beta}^{0}(\Omega)$ such that

$$
\mid \begin{aligned}
& \mathcal{A}_{\beta} v=-\sigma_{+} \Delta v \\
& D\left(\mathcal{A}_{\beta}\right):=\mathrm{V}_{\beta}^{2}(\Omega) \cap \stackrel{\circ}{\mathrm{V}}_{\beta-1}^{1}(\Omega) .
\end{aligned}
$$

The proof of the following classical result can be found in the original paper [22] or, for example, in [36, Chap 2], [29, Chap 1].

Proposition 2.1. If $\beta \in(1 / 2 ; 3 / 2)$ then $\mathcal{A}_{\beta}$ is an isomorphism.

\section{$2.2 \quad$ Near field operator}

Introduce the rapid coordinate $\boldsymbol{\xi}:=\delta^{-1} \boldsymbol{x}$ and let $\delta$ tend to zero. Then, define the function $\sigma^{\infty}: \mathbb{R}^{3} \rightarrow \mathbb{R}$ such that $\sigma^{\infty}=\sigma_{+}$in $\Xi_{+}:=\mathbb{R}^{3} \backslash \Xi_{-}$and $\sigma^{\infty}=\sigma_{-}$in $\Xi_{-}$. In the sequel, the following near field operator $\mathrm{B}^{\infty}: D\left(\mathrm{~B}^{\infty}\right) \rightarrow \mathrm{L}^{2}\left(\mathbb{R}^{3}\right)$ will play a key role in the analysis:

$$
\mid \begin{aligned}
& \mathrm{B}^{\infty} w=-\operatorname{div}\left(\sigma^{\infty} \nabla w\right) \\
& D\left(\mathrm{~B}^{\infty}\right):=\left\{w \in \mathrm{H}^{1}\left(\mathbb{R}^{3}\right) \mid \operatorname{div}\left(\sigma^{\infty} \nabla w\right) \in \mathrm{L}^{2}\left(\mathbb{R}^{3}\right)\right\} .
\end{aligned}
$$

The description of $D\left(\mathrm{~B}^{\infty}\right)$ is less classical than the one of $D\left(\mathrm{~A}^{0}\right)$ because the sign of the parameter $\sigma^{\infty}$ is not constant on $\mathbb{R}^{3}$. Nevertheless, when $\kappa_{\sigma} \neq-1$, $\mathrm{B}^{\infty}$ has elliptic regularity properties. In the next result, we consider the norm $\|w\|_{\mathrm{H}^{2}\left(\mathbb{R}^{3} \backslash \Gamma\right)}:=\|w\|_{\mathrm{H}^{2}\left(\Xi_{-}\right)}+\|w\|_{\mathrm{H}^{2}\left(\Xi_{+}\right)}$, as well as the jump of normal derivative $\left[\sigma \partial_{\boldsymbol{n}} w\right]_{\Gamma}:=\left.\sigma_{+} \partial_{\boldsymbol{n}} w\right|_{\Gamma} ^{+}-\left.\sigma_{-} \partial_{\boldsymbol{n}} w\right|_{\Gamma} ^{-}$, where $\boldsymbol{n}$ denotes the unit outward normal vector to $\Gamma$ directed from $\Xi_{-}$to $\Xi_{+}$.

Proposition 2.2. Assume that $\kappa_{\sigma} \neq-1$. Then any $w \in D\left(\mathrm{~B}^{\infty}\right)$ satisfies $w \in \mathrm{H}^{2}\left(\Xi_{ \pm}\right)$and $\left[\sigma \partial_{\boldsymbol{n}} w\right]_{\Gamma}=$ 0 . Moreover, there exists a constant $C>0$ such that

$$
\|w\|_{\mathrm{H}^{2}\left(\mathbb{R}^{3} \backslash \Gamma\right)} \leq C\left(\left\|\operatorname{div}\left(\sigma^{\infty} \nabla w\right)\right\|_{\mathbb{R}^{3}}+\|w\|_{\mathbb{R}^{3}}\right), \quad \forall w \in D\left(\mathrm{~B}^{\infty}\right) .
$$


Proof. Clearly, any element $w \in \mathrm{H}^{1}\left(\mathbb{R}^{3}\right)$ that verifies $w \in \mathrm{H}^{2}\left(\Xi_{ \pm}\right)$and $\left[\sigma \partial_{\boldsymbol{n}} w\right]_{\Gamma}=0$ belongs to $D\left(\mathrm{~B}^{\infty}\right)$. Now, let us pick some $w \in D\left(\mathrm{~B}^{\infty}\right)$. The relation $\left[\sigma \partial_{n} w\right]_{\Gamma}=0$ is classically obtained using Green's formula. Now, we wish to prove (11). Let $\psi$ be the cut-off function defined in (4). There holds $\operatorname{div}\left(\sigma^{\infty} \nabla(\psi w)\right)=f$ with $f=\psi \operatorname{div}\left(\sigma^{\infty} \nabla w\right)+2 \sigma_{+} \nabla \psi \cdot \nabla w+\sigma_{+} w \Delta \psi \in \mathrm{L}^{2}\left(\mathbb{R}^{3}\right)$ (notice that $\sigma^{\infty}=\sigma_{+}$ on the supports of $\nabla \psi$ and $\Delta \psi$ ). Since $\kappa_{\sigma} \neq-1$ and since $\Gamma=\partial \Xi_{-}$is smooth, the results of [23, 44] allow to prove that $\left.\psi w\right|_{\Xi_{ \pm}}$belongs to $\mathrm{H}^{2}\left(\Xi_{ \pm}\right)$with the estimate $\|\psi w\|_{\mathrm{H}^{2}\left(\Xi_{ \pm}\right)} \leq C\left(\|f\|_{\mathbb{R}^{3}}+\|w\|_{\Omega}\right)$. Here and in the sequel, $C>0$ denotes a constant independent of $w$ which can change from one line to another. Using interior regularity in $\Xi_{+}$, one establishes with classical techniques (see [23]) that $\|f\|_{\mathbb{R}^{3}} \leq C\left(\left\|\psi \operatorname{div}\left(\sigma^{\infty} \nabla w\right)\right\|_{\mathbb{R}^{3}}+\|w\|_{\Omega}\right)$. This implies

$$
\|\psi w\|_{\mathrm{H}^{2}\left(\Xi_{ \pm}\right)} \leq C\left(\left\|\psi \operatorname{div}\left(\sigma^{\infty} \nabla w\right)\right\|_{\mathbb{R}^{3}}+\|w\|_{\Omega}\right), \quad \forall w \in D\left(\mathrm{~B}^{\infty}\right),
$$

with a constant $C>0$ that does not depend on $w$. It remains to estimate $\operatorname{div}\left(\sigma^{\infty} \nabla(\chi w)\right)=\sigma_{+} \Delta(\chi w)$. Thanks to Plancherel's theorem, we can write $\|\chi w\|_{\mathrm{H}^{2}\left(\Xi_{+}\right)} \leq C\left(\left\|\operatorname{div}\left(\sigma^{\infty} \nabla(\chi w)\right)\right\|_{\Xi_{+}}+\|\chi w\|_{\mathbb{R}^{3}}\right)$. Using again interior regularity in $\Xi_{+}$, we deduce that $\|\chi w\|_{\mathrm{H}^{2}\left(\Xi_{+}\right)} \leq C\left(\left\|\chi \operatorname{div}\left(\sigma^{\infty} \nabla w\right)\right\|_{\mathbb{R}^{3}}+\|w\|_{\mathbb{R}^{3}}\right)$. This last inequality together with (12) finally leads to (11) since $\psi+\chi=1$.

Let us describe the spectrum $\mathfrak{S}\left(\mathrm{B}^{\infty}\right)$ of the operator $\mathrm{B}^{\infty}$ when $\kappa_{\sigma} \neq-1$. According to the 3D version of [5, Thm 5.2], for all $f \in \mathrm{L}^{2}\left(\mathbb{R}^{3}\right)$, we know that the problem "find $v \in \mathrm{H}^{1}\left(\mathbb{R}^{3}\right)$ such that $\left(\sigma^{\infty} \nabla v, \nabla v^{\prime}\right)_{\mathbb{R}^{3}} \pm i\left(v, v^{\prime}\right)_{\mathbb{R}^{3}}=\left(f, v^{\prime}\right)_{\mathbb{R}^{3}}$ for all $v^{\prime} \in \mathrm{H}^{1}\left(\mathbb{R}^{3}\right)$ ", has a unique solution. On the other hand, since $-\sigma_{+} \Delta \pm i \mathrm{Id}: \mathrm{H}^{2}\left(\mathbb{R}^{3}\right) \rightarrow \mathrm{L}^{2}\left(\mathbb{R}^{3}\right)$ is bijective, we can prove that $\mathrm{B}^{\infty} \pm i$ Id is bijective. Here, Id denotes the identity of $\mathrm{L}^{2}\left(\mathbb{R}^{3}\right)$. From [4, Thm 4.1.7], we conclude that $\mathrm{B}^{\infty}$ is self-adjoint. Moreover, observing that $\sigma^{\infty}=\sigma_{+}$outside the compact region $\bar{\Xi}_{-}$, using again the 3D version of [5, Thm 5.2], we can show that $\mathrm{B}^{\infty}$ has the same continuous spectrum as the Laplace operator in $\mathbb{R}^{3}$. In other words, there holds $\mathfrak{S}_{c}\left(\mathrm{~B}^{\infty}\right)=[0 ;+\infty)$. The interval $(-\infty ; 0)$ contains discrete spectrum only. Working as in [7, Prop 4.1], we can build a sequence $\left(\zeta_{n}\right)_{n}$ of elements of $D\left(\mathrm{~B}^{\infty}\right)$ such that $\lim _{n \rightarrow \infty}\left(\mathrm{B}^{\infty} \zeta_{n}, \zeta_{n}\right)_{\mathbb{R}^{3}}=-\infty$ and $\left\|\zeta_{n}\right\|_{\mathbb{R}^{3}}=1$. According to [4, Cor 4.1.5], this proves that the discrete spectrum of $\mathrm{B}^{\infty}$ is equal to a sequence of eigenvalues

$$
\mathfrak{S}\left(\mathrm{B}^{\infty}\right) \backslash \overline{\mathbb{R}_{+}}=\left\{\mu_{-n}\right\}_{n \geq 1} \quad \text { with } \quad 0>\mu_{-1} \geq \mu_{-2} \geq \cdots \geq \mu_{-n} \ldots \underset{n \rightarrow+\infty}{\rightarrow}-\infty
$$

where each eigenvalue is repeated according to its multiplicity. In the following proposition, we establish that the eigenfunctions corresponding to the negative spectrum of $\mathrm{B}^{\infty}$ are localized: they decay exponentially at infinity.

Proposition 2.3. Assume that $\kappa_{\sigma} \neq-1$. For any eigenfunction $w$ of $\mathrm{B}^{\infty}$ associated with an eigenvalue $\mu \in \mathfrak{S}\left(\mathrm{B}^{\infty}\right) \backslash \overline{\mathbb{R}_{+}}$satisfying $\|w\|_{\mathbb{R}^{3}}=1$, we have

$$
\int_{\mathbb{R}^{3}}\left(|w(\boldsymbol{\xi})|^{2}+|\nabla w(\boldsymbol{\xi})|^{2}\right) \exp \left(|\boldsymbol{\xi}| \sqrt{|\mu| / \sigma_{+}}\right) d \boldsymbol{\xi}<+\infty .
$$

Proof. The proof of this proposition will rely on a technique used in $[19,10,33]$. Consider some $\gamma>0$ whose appropriate value will be fixed later on, and pick some $T \geq 2$. We introduce the weight function $\mathscr{W}_{\gamma}^{T}$ such that

$$
\mathscr{W}_{\gamma}^{T}(\boldsymbol{\xi})=\left\{\begin{array}{llr}
\exp (\gamma) & \text { for } & |\boldsymbol{\xi}| \leq 1 \\
\exp (\gamma|\boldsymbol{\xi}|) & \text { for } & 1 \leq|\boldsymbol{\xi}| \leq T \\
\exp (\gamma T) & \text { for } & |\boldsymbol{\xi}| \geq T
\end{array}\right.
$$

It is clear that $\mathscr{W}_{\gamma}^{T}$ is bounded and continuous. If $w$ is an eigenfunction of $\mathrm{B}^{\infty}$ associated with the eigenvalue $\mu<0$, there holds $\left(\sigma^{\infty} \nabla w, \nabla w^{\prime}\right)_{\mathbb{R}^{3}}=\mu\left(w, w^{\prime}\right)_{\mathbb{R}^{3}}$ for all $w^{\prime} \in \mathrm{H}^{1}\left(\mathbb{R}^{3}\right)$. Choosing $w^{\prime}=\left(\mathscr{W}_{\gamma}^{T}\right)^{2} w$ (observe that this function is indeed an element of $\mathrm{H}^{1}\left(\mathbb{R}^{3}\right)$ ), we obtain

$$
\begin{aligned}
|\mu|\left\|\mathscr{W}_{\gamma}^{T} w\right\|_{\mathbb{R}^{3}}^{2} & =-\left(\sigma^{\infty} \nabla w, \nabla\left(\left(\mathscr{W}_{\gamma}^{T}\right)^{2} w\right)\right)_{\mathbb{R}^{3}} \\
& =-\left(\sigma^{\infty} \mathscr{W}_{\gamma}^{T} \nabla w, \nabla\left(\mathscr{W}_{\gamma}^{T} w\right)\right)_{\mathbb{R}^{3}}-\left(\sigma^{\infty} \mathscr{W}_{\gamma}^{T} \nabla w, w \nabla \mathscr{W}_{\gamma}^{T}\right)_{\mathbb{R}^{3}} \\
& =-\left(\sigma^{\infty}\left(\nabla\left(\mathscr{W}_{\gamma}^{T} w\right)-w \nabla \mathscr{W}_{\gamma}^{T}\right), \nabla\left(\mathscr{W}_{\gamma}^{T} w\right)\right)_{\mathbb{R}^{3}}-\left(\sigma^{\infty} \mathscr{W}_{\gamma}^{T} \nabla w, w \nabla \mathscr{W}_{\gamma}^{T}\right)_{\mathbb{R}^{3}} \\
& =-\left(\sigma^{\infty} \nabla\left(\mathscr{W}_{\gamma}^{T} w\right), \nabla\left(\mathscr{W}_{\gamma}^{T} w\right)\right)_{\mathbb{R}^{3}}+\left(\sigma^{\infty} w \nabla \mathscr{W}_{\gamma}^{T}, w \nabla \mathscr{W}_{\gamma}^{T}\right)_{\mathbb{R}^{3}} .
\end{aligned}
$$


On $\Xi_{-}$, we have $\mathscr{W}_{\gamma}^{T}=\exp (\gamma)$. Therefore, (15) rewrites as

$$
|\mu|\left\|\mathscr{W}_{\gamma}^{T} w\right\|_{\mathbb{R}^{3}}^{2}+\sigma_{+}\left\|\nabla\left(\mathscr{W}_{\gamma}^{T} w\right)\right\|_{\Xi_{+}}^{2}=\exp (2 \gamma)\left|\sigma_{-}\right|\|\nabla w\|_{\Xi_{-}}^{2}+\sigma_{+}\left\|w \nabla \mathscr{W}_{\gamma}^{T}\right\|_{\mathbb{R}^{3}}^{2}
$$

Add $\sigma_{+}\left\|\nabla\left(\mathscr{W}_{\gamma}^{T} w\right)\right\|_{\Xi_{-}}^{2}=\sigma_{+} \exp (2 \gamma)\|\nabla w\|_{\Xi_{-}}^{2}$ on each side of (16), and use triangular inequality $\left\|\nabla\left(\mathscr{W}_{\gamma}^{T} w\right)\right\|_{\mathbb{R}^{3}}^{2} \geq \frac{1}{2}\left\|\mathscr{W}_{\gamma}^{T} \nabla w\right\|_{\mathbb{R}^{3}}^{2}-\left\|w \nabla \mathscr{W}_{\gamma}^{T}\right\|_{\mathbb{R}^{3}}^{2}$, to obtain

$$
|\mu|\left\|w \mathscr{W}_{\gamma}^{T}\right\|_{\mathbb{R}^{3}}^{2}-2 \sigma_{+}\left\|w \nabla \mathscr{W}_{\gamma}^{T}\right\|_{\mathbb{R}^{3}}^{2}+\frac{\sigma_{+}}{2}\left\|\mathscr{W}_{\gamma}^{T} \nabla w\right\|_{\mathbb{R}^{3}}^{2} \leq \exp (2 \gamma)\left(\sigma_{+}+\left|\sigma_{-}\right|\right)\|\nabla w\|_{\Xi_{-}}^{2} .
$$

We have $\left|\nabla \mathscr{W}_{\gamma}^{T}\right| \leq \gamma\left|\mathscr{W}_{\gamma}^{T}\right|$, which implies $|\mu|\left\|w \mathscr{W}_{\gamma}^{T}\right\|_{\mathbb{R}^{3}}^{2}-2 \sigma_{+}\left\|w \nabla \mathscr{W}_{\gamma}^{T}\right\|_{\mathbb{R}^{3}}^{2} \geq\left(|\mu|-2 \gamma^{2} \sigma_{+}\right)\left\|w \mathscr{W}_{\gamma}^{T}\right\|_{\mathbb{R}^{3}}^{2}$. From this, we conclude that

$$
|\mu|\left\|\mathscr{W}_{\gamma}^{T} w\right\|_{\mathbb{R}^{3}}^{2}+\sigma_{+}\left\|\mathscr{W}_{\gamma}^{T} \nabla w\right\|_{\mathbb{R}^{3}}^{2} \leq 2 \exp (2 \gamma)\left(\sigma_{+}+\left|\sigma_{-}\right|\right)\|\nabla w\|_{\Xi_{-}}^{2}, \quad \text { for } \gamma=\frac{1}{2} \sqrt{|\mu| / \sigma_{+}} .
$$

There only remains to let $T$ tend to $+\infty$. Since the right-hand side of the inequality above is independent of $T$, this concludes the proof.

In the sequel, we will need to work with an operator analog to $\mathrm{B}^{\infty}$ but considered in Sobolev spaces with weight at infinity. If $\boldsymbol{\xi} \in \mathbb{R}^{3}$, we denote $\rho:=|\boldsymbol{\xi}|$. For $\beta \in \mathbb{R}, k \geq 0$, we introduce the space $\mathcal{V}_{\beta}^{k}\left(\mathbb{R}^{3}\right)$ defined as the completion of the set $\mathscr{C}_{0}^{\infty}\left(\mathbb{R}^{3}\right)$ in the norm

$$
\|w\|_{\mathcal{V}_{\beta}^{k}\left(\mathbb{R}^{3}\right)}:=\left(\sum_{|\alpha| \leq k} \int_{\mathbb{R}^{3}}(1+\rho)^{2(\beta+|\alpha|-k)}\left|\partial_{\xi}^{\alpha} w\right|^{2} d \boldsymbol{\xi}\right)^{1 / 2} .
$$

We also define $\mathcal{V}_{\beta}^{k}\left(\Xi_{ \pm}\right):=\left\{\left.w\right|_{\Xi_{ \pm}} \mid w \in \mathcal{V}_{\beta}^{k}\left(\mathbb{R}^{3}\right)\right\}$ (although $\mathcal{V}_{\beta}^{k}\left(\Xi_{-}\right)=\mathrm{H}^{k}\left(\Xi_{-}\right.$) for all $\beta \in \mathbb{R}$, as $\Xi_{-}$is bounded). Introduce the operator $\mathcal{B}_{\beta}: D\left(\mathcal{B}_{\beta}\right) \rightarrow \mathcal{V}_{\beta}^{0}\left(\mathbb{R}^{3}\right)$ such that

$$
\mid \begin{aligned}
& \mathcal{B}_{\beta} w=-\operatorname{div}\left(\sigma^{\infty} \nabla w\right) \quad \forall w \in D\left(\mathcal{B}_{\beta}\right) \\
& D\left(\mathcal{B}_{\beta}\right):=\left\{w \in \mathcal{V}_{\beta-1}^{1}\left(\mathbb{R}^{3}\right) \mid \operatorname{div}\left(\sigma^{\infty} \nabla w\right) \in \mathcal{V}_{\beta}^{0}\left(\mathbb{R}^{3}\right)\right\} .
\end{aligned}
$$

Working as in the proof of Proposition 2.2 and using the Kondratiev theory, one can show that any element $w \in D\left(\mathcal{B}_{\beta}\right)$ verifies $w \in \mathrm{V}_{\beta}^{2}\left(\Xi_{ \pm}\right)$and $\left[\sigma \partial_{\boldsymbol{n}} w\right]_{\Gamma}=0$. Conversely it is straightforward to check that any $w \in \mathrm{V}_{\beta-1}^{1}\left(\Xi_{ \pm}\right)$satisfying these two conditions belongs to $D\left(\mathcal{B}_{\beta}\right)$. Hence $D\left(\mathcal{B}_{\beta}\right)$ is a closed subset of $\mathcal{V}_{\beta}^{2}\left(\mathbb{R}^{3} \backslash \Gamma\right)$, and $\mathcal{B}_{\beta}$ is continuous when equipping $D\left(\mathcal{B}_{\beta}\right)$ with the norm $\|w\|_{\mathcal{V}_{\beta}^{2}\left(\mathbb{R}^{3} \backslash \Gamma\right)}:=$ $\|w\|_{\mathcal{V}_{\beta}^{2}\left(\Xi_{+}\right)}+\|w\|_{\mathcal{V}_{\beta}^{2}\left(\Xi_{-}\right)}$.

Proposition 2.4. Assume that $\kappa_{\sigma} \neq-1$ and that $\beta \in(1 / 2 ; 3 / 2)$. Then $\mathcal{B}_{\beta}$ is a Fredholm operator with $\operatorname{ind}\left(\mathcal{B}_{\beta}\right):=\operatorname{dim}\left(\operatorname{ker} \mathcal{B}_{\beta}\right)-\operatorname{dim}\left(\operatorname{coker} \mathcal{B}_{\beta}\right)=0^{1}$.

Proof. Once the difficulty of the change of sign of $\sigma^{\infty}$ on the compact set $\Xi_{-}$has been tackled thanks to $[14,5]$, the proof of this proposition is rather classical and we will just sketch it. From the Kondratiev theory [22], we know that for $\beta \in(1 / 2 ; 3 / 2)$, the Laplace operator maps isomorphically $\mathcal{V}_{\beta}^{2}\left(\mathbb{R}^{3}\right)$ onto $\mathcal{V}_{\beta}^{0}\left(\mathbb{R}^{3}\right)$. Using this result and inequality (12), we obtain

$$
\|w\|_{\mathcal{V}_{\beta}^{2}\left(\mathbb{R}^{3} \backslash \Gamma\right)} \leq C\left(\left\|\operatorname{div}\left(\sigma^{\infty} \nabla w\right)\right\|_{\mathcal{V}_{\beta}^{0}\left(\mathbb{R}^{3}\right)}+\|w\|_{\Omega}\right), \quad \forall w \in D\left(\mathcal{B}_{\beta}\right) .
$$

Since the map $\left.w \mapsto w\right|_{\Omega}$ from $D\left(\mathcal{B}_{\beta}\right)$ to $\mathrm{L}^{2}(\Omega)$ is compact, using the classical extension [46] of the well-known Peetre's lemma [40] (see also lemma 5.1 of [23, Chap 2]), we infer from the previous $a$ priori estimate that $\mathcal{B}_{\beta}$ has a kernel of finite dimension and that its range is closed in $\mathcal{V}_{\beta}^{0}\left(\mathbb{R}^{3}\right)$. As a consequence of the latter property, $\left[4\right.$, Thm 3.3.5] ensures that coker $\mathcal{B}_{\beta}$ is isomorphic to ker $\mathcal{B}_{\beta}{ }^{*}$ where $\mathcal{B}_{\beta}{ }^{*}$ denotes the adjoint of $\mathcal{B}_{\beta}$. But the adjoint of $\mathcal{B}_{\beta}$ is the operator $\mathcal{B}_{2-\beta}$, which has a kernel of finite dimension because for $\beta \in(1 / 2 ; 3 / 2), 2-\beta$ also belongs to $(1 / 2 ; 3 / 2)$. Therefore coker $\mathcal{B}_{\beta}$ is of finite dimension. Using the Kondratiev theory again, we can establish that for all $\beta \in(1 / 2 ; 3 / 2)$, there holds ker $\mathcal{B}_{\beta}=\cap_{\gamma \in(1 / 2 ; 3 / 2)} \operatorname{ker} \mathcal{B}_{\gamma}$. This implies $\operatorname{ker} \mathcal{B}_{\beta}=\operatorname{ker} \mathcal{B}_{2-\beta}, \operatorname{dim}\left(\operatorname{coker} \mathcal{B}_{\beta}\right)=\operatorname{dim}\left(\operatorname{ker} \mathcal{B}_{2-\beta}\right)=$ $\operatorname{dim}\left(\operatorname{ker} \mathcal{B}_{\beta}\right)$ and so ind $\left(\mathcal{B}_{\beta}\right)=0$.

\footnotetext{
${ }^{1}$ We recall that if $\mathrm{X}, \mathrm{Y}$ are two Banach spaces and if $L: \mathrm{X} \rightarrow \mathrm{Y}$ is a continuous linear map with closed range, then the cokernel of $L$ is defined as coker $L:=(\mathrm{Y} /$ range $L)$.
} 
Depending on the parameter $\sigma_{ \pm}$and on the domain $\Xi_{-}$, it can happen that the operator $\mathcal{B}_{\beta}$ gets a non trivial (finite dimensional) kernel. We discard this possibility, considering an additional assumption

Assumption 1. There exists $\beta \in(1 / 2 ; 3 / 2)$ such that the operator $\mathcal{B}_{\beta}$ is injective.

According to Proposition 2.4 and the Kondratiev theory [22], Assumption 1 implies that $\mathcal{B}_{\beta}: D\left(\mathcal{B}_{\beta}\right) \rightarrow$ $\mathcal{V}_{\beta}^{0}\left(\mathbb{R}^{3}\right)$ is an isomorphism for all $\beta \in(1 / 2,3 / 2)$ (and not just for one value of $\beta$ ). For a concrete case where this assumption is satisfied, one may for example consider the situation of Section 6 .

Remark 2.1. This assumption is interesting in its own and there exist results to check whether or not it holds for a given configuration. It is related to a question investigated by $H$. Poincaré in [42]. As it is done in the introduction of the very interesting paper [21], let us summarize Poincaré's problem with our notation. Let $w: \mathbb{R}^{3} \rightarrow \mathbb{R}$ be a continuous function whose restrictions to $\Xi_{ \pm}$are harmonic (like the elements of the kernel of $\left.\mathcal{B}_{\beta}\right)$. If we impose the total energy $\|\nabla w\|_{\mathbb{R}^{3}}$ to be equal to one, what is the minimum of $\|\nabla w\|_{\Xi_{-}}$? The answer is simple: this minimum is zero and it is attained for $w=w^{0}$ where $w^{0}=1$ in $\Xi_{-}$. Now, if we assume that $w$ satisfies both $\|\nabla w\|_{\mathbb{R}^{3}}=1$ and the orthogonality relation $\left(\nabla w, \nabla w^{0}\right)_{\mathbb{R}^{3}}=0$, what is the minimum of $\|\nabla w\|_{\Xi_{-}}$? Is it attained? It turns out that the minimum is indeed attained and is equal to some $m^{1}>0$. Moreover, if $w^{1}$ is a function which realizes this minimum, then there holds $\left.\partial_{\boldsymbol{n}} w^{1}\right|_{\Gamma} ^{+}=-\left.m^{1} \partial_{\boldsymbol{n}} w^{1}\right|_{\Gamma} ^{-}$on $\partial \Xi_{-}$. In other words, $w^{1}$ belongs to the kernel of $\mathcal{B}_{\beta}$ when the contrast $\kappa_{\sigma}=\sigma_{-} / \sigma_{+}$verifies $\kappa_{\sigma}=-m^{1}$. Continuing the process and imposing the orthogonality relations $\left(\nabla w, \nabla w^{0}\right)_{\mathbb{R}^{3}}=0,\left(\nabla w, \nabla w^{1}\right)_{\mathbb{R}^{3}}=0 \ldots$, we can express all the values of the contrasts $\kappa_{\sigma}$ for which $\mathcal{B}_{\beta}$ fails to be injective in term of the extrema of the ratios of energies $\|\nabla w\|_{\Xi_{-}} /\|\nabla w\|_{\mathbb{R}^{3}}$. For more details concerning this question, we refer the reader to [21, 18, 41, 8, 17].

Remark 2.2. In the physical literature [25], values of $\kappa_{\sigma}=\sigma_{-} / \sigma_{+}$leading to a non-trivial kernel for $\mathcal{B}_{\beta}$ are referred to as plasmonic eigenvalues. Note that, although there is a growing interest on this so-called plasmonic eigenvalue problem, the latter is not the concern of the present article. Here we assume that $\kappa_{\sigma}$ is fixed and not a plasmonic eigenvalue for the operator $\mathcal{B}_{\beta}$ (see Assumption 1 above), and we study the spectrum of the corresponding operator $\mathrm{A}^{\delta}$.

Remark 2.3. The study of the asymptotics of the eigenvalues of $\mathrm{A}^{\delta}$ as $\delta$ goes to zero remains an open question when Assumption 1 does not hold.

In this paper, we focus on the behaviour of eigenvalues and we leave aside the justification of asymptotics of eigenfunctions. We shall prove that if Assumption 1 holds, then for all $n \in \mathbb{N}^{*}:=\{1,2, \ldots\}$, the eigenvalue $\lambda_{n}^{\delta} \geq 0$ of $\mathrm{A}^{\delta}$ converges to the corresponding eigenvalue $\mu_{n}>0$ of $\mathrm{A}^{0}$. We shall also show that for all $n \in \mathbb{N}^{*}$, the eigenvalue $\lambda_{-n}^{\delta}<0$ of $\mathrm{A}^{\delta}$ is such that $\delta^{2} \lambda_{-n}$ converges to $\mu_{-n}<0$, where $\mu_{-n}<0$ is the eigenvalue of $\mathrm{B}^{\infty}$ defined in (13). Before doing that, in the next section, we investigate the source term problem associated with the original spectral Problem (1). In the process, we will establish a stability estimate which will reveal useful for the study of the initial problem.

\section{Asymptotic analysis for the source term problem}

Let $f$ be a source term in $\mathrm{L}^{2}(\Omega)$. In this section, we consider the problem

$$
\mid \begin{aligned}
& \text { Find } u^{\delta} \in \mathrm{H}_{0}^{1}(\Omega) \text { such that } \\
& -\operatorname{div}\left(\sigma^{\delta} \nabla u^{\delta}\right)=f \quad \text { in } \Omega .
\end{aligned}
$$

For a fixed $\delta \in(0 ; 1]$, using Proposition 2.1 and the 3D version of [5, Thm 5.2], we can show, working as in the proof of Proposition 2.4, that Problem (20) is uniquely solvable if and only if it is injective. Under Assumption 1, when the contrast satisfies $\kappa_{\sigma} \neq-1$, we will establish that it is injective (so that $\left(\mathrm{A}^{\delta}\right)^{-1}$ is well-defined) for $\delta$ small enough. Then, we provide, and justify with an error estimate, an asymptotic expansion of the solution $u^{\delta}$. 


\subsection{A stability estimate}

We start by proving an important stability estimate for problem (20). Because the sign of $\sigma^{\delta}$ changes on $\Omega$, this is a delicate procedure and the variational approach developed in $[6,5]$ to establish Fredholm property for (20) seems useless here. Instead, we will employ a method introduced in [29, Chap 2], [31] (see also [13] for an example of application in the context of negative materials), relying on the use of overlapping cut-off functions. To implement this technique, we need to introduce the Hilbert spaces $\mathrm{V}_{\beta, \delta}^{k}(\omega), k \in \mathbb{N}, \beta \in \mathbb{R}$, over a domain $\omega \subset \mathbb{R}^{3}$. These spaces are defined as the completions of $\mathscr{C}^{\infty}(\bar{\omega})$ for the weighted norms

$$
\|v\|_{\mathrm{V}_{\beta, \delta}^{k}(\omega)}:=\left(\sum_{|\alpha| \leq k} \int_{\Omega}(r+\delta)^{2(\beta+|\alpha|-k)}\left|\partial_{x}^{\alpha} v\right|^{2} d \boldsymbol{x}\right)^{1 / 2} .
$$

Observe that for any $\beta \in \mathbb{R}$ and $\delta>0$, the space $\mathrm{V}_{\beta, \delta}^{k}(\Omega)$ coincides with $\mathrm{H}^{k}(\Omega)$ because the norm (21) is equivalent to $\|\cdot\|_{\mathrm{H}^{k}(\omega)}$. However, the constants coming into play in this equivalence definitely depend on $\delta$ which is a crucial feature. In the sequel, we shall consider the norm defined by $\|v\|_{\mathrm{V}_{\beta, \delta}^{2}\left(\Omega \backslash \Gamma^{\delta}\right)}:=$ $\|v\|_{\mathrm{V}_{\beta, \delta}^{2}\left(\Omega_{+}^{\delta}\right)}+\|v\|_{\mathrm{V}_{\beta, \delta}^{2}\left(\Omega_{-}^{\delta}\right)}$.

Proposition 3.1. Assume that $\kappa_{\sigma} \neq-1$ and that Assumption 1 holds. Then there is some $\delta_{0}>0$ such that the operator $\mathrm{A}^{\delta}: D\left(\mathrm{~A}^{\delta}\right) \rightarrow \mathrm{L}^{2}(\Omega)$ defined by (2) is an isomorphism for all $\delta \in\left(0 ; \delta_{0}\right]$. Moreover, there holds $v \in \mathrm{H}^{2}\left(\Omega_{ \pm}^{\delta}\right)$ for all $v \in D\left(\mathrm{~A}^{\delta}\right)$ and, for any $\beta \in(1 / 2 ; 3 / 2)$, there exists $C_{\beta}>0$ independent of $\delta$ such that

$$
C_{\beta} \leq \inf _{v \in D\left(\mathrm{~A}^{\delta}\right) \backslash\{0\}} \frac{\left\|\mathrm{A}^{\delta} v\right\|_{\mathrm{V}_{\beta, \delta}^{0}(\Omega)}}{\|v\|_{\mathrm{V}_{\beta, \delta}^{2}\left(\Omega \backslash \Gamma^{\delta}\right)}}, \quad \forall \delta \in\left(0 ; \delta_{0}\right] .
$$

Proof. We will construct explicitly the inverse of $\mathrm{A}^{\delta}$. We first define a linear map $\mathrm{R}^{\delta}: \mathrm{L}^{2}(\Omega) \rightarrow D\left(\mathrm{~A}^{\delta}\right)$ in the following manner. Take any element $f \in \mathrm{L}^{2}(\Omega)$. Decompose it in an inner and an outer contribution,

$$
f(\boldsymbol{x})=g(\boldsymbol{x})+G(\boldsymbol{x} / \delta) \quad \text { with } \quad g(\boldsymbol{x})=\chi_{\sqrt{\delta}}(r) f(\boldsymbol{x}) \quad \text { and } \quad G(\boldsymbol{\xi})=\psi_{\sqrt{\delta}}(\delta \rho) f(\delta \boldsymbol{\xi}) .
$$

Let us emphasize that according to the definition of $\chi, \psi$ (see (5)), there holds $\chi_{\sqrt{\delta}}+\psi_{\sqrt{\delta}}=1$. Moreover, since $\psi_{\sqrt{\delta}}=1$ for $r \leq \sqrt{\delta}$ and $\psi_{\sqrt{\delta}}=0$ for $r \geq 2 \sqrt{\delta}, \operatorname{supp}(G)$ (the support of $G$ ) is bounded in $\mathbb{R}^{3}$. As a consequence, for all $\varepsilon \in \mathbb{R}, \delta>0$, we have $g \in \mathrm{V}_{\beta-\varepsilon, \delta}^{0}(\Omega)$ and $G \in \mathrm{V}_{\beta+\varepsilon}^{0}\left(\mathbb{R}^{3}\right)$. Besides, for $\varepsilon \geq 0$, the following estimates are valid:

$$
\begin{aligned}
& \|g\|_{\mathrm{V}_{\beta-\varepsilon, \delta}^{0}(\Omega)}=\left\|(\delta+r)^{\beta-\varepsilon} g\right\|_{\Omega \backslash \overline{\mathrm{B}(O, \sqrt{\delta})}} \leq C \delta^{-\varepsilon / 2}\left\|(\delta+r)^{\beta} g\right\|_{\Omega \backslash \overline{\mathrm{B}(O, \sqrt{\delta})}} \leq \delta^{-\varepsilon / 2}\|f\|_{\mathrm{V}_{\beta, \delta}^{0}(\Omega)} \\
& \text { and }\|G\|_{\mathcal{V}_{\beta+\varepsilon}^{0}\left(\mathbb{R}^{3}\right)}=\left\|(1+\rho)^{\beta+\varepsilon} G\right\|_{\mathrm{B}(O, 2 / \sqrt{\delta})} \\
& \leq C \delta^{-\varepsilon / 2}\left\|(1+\rho)^{\beta} G\right\|_{\mathrm{B}(O, 2 / \sqrt{\delta})} \leq C \delta^{-\beta-3 / 2-\varepsilon / 2}\|f\|_{\mathrm{V}_{\beta, \delta}^{0}(\Omega)} .
\end{aligned}
$$

Here and in the sequel, $C>0$ denotes a constant, which can change from one line to another, depending on $\beta, \varepsilon$ but not on $\delta$. The last inequality in (24) has been obtained making the change of variables $\boldsymbol{x}=\delta \boldsymbol{\xi}$. This explains the appearance of the term $\delta^{-3 / 2-\beta}$. Let us choose $\varepsilon>0$ small enough so that $[\beta-\varepsilon ; \beta+\varepsilon] \in(1 / 2 ; 3 / 2)$. Let us consider $v \in D\left(\mathcal{A}_{\beta-\varepsilon}\right), V \in D\left(\mathcal{B}_{\beta+\varepsilon}\right)$ such that

$$
\mathcal{A}_{\beta-\varepsilon} v=g, \quad \mathcal{B}_{\beta+\varepsilon} V=\delta^{2} G,
$$

where $\mathcal{A}_{\beta-\varepsilon}$ and $\mathcal{B}_{\beta+\varepsilon}$ were introduced respectively in (9) and (19). Proposition 2.1 and Proposition 2.4 coupled with Assumption 1 ensure that $v, V$ are well-defined and that they satisfy the estimates

$$
\|v\|_{\mathrm{V}_{\beta-\varepsilon}^{2}(\Omega)} \leq C\|g\|_{\mathrm{V}_{\beta-\varepsilon}^{0}(\Omega)} \leq C\|g\|_{\mathrm{V}_{\beta-\varepsilon, \delta}^{0}(\Omega)}, \quad\|V\|_{\mathcal{V}_{\beta+\varepsilon}^{2}\left(\mathbb{R}^{3} \backslash \Gamma\right)} \leq C \delta^{2}\|G\|_{\mathcal{V}_{\beta+\varepsilon}^{0}\left(\mathbb{R}^{3}\right)} .
$$

Finally, we define the operator $\mathrm{R}^{\delta}$ such that for all $f \in \mathrm{L}^{2}(\Omega), \mathrm{R}^{\delta} f=\hat{u}^{\delta}$ with

$$
\hat{u}^{\delta}(\boldsymbol{x}):=\chi_{\delta}(r) v(\boldsymbol{x})+\psi(r) V_{\delta}(\boldsymbol{x}), \quad \text { where } \quad V_{\delta}(\boldsymbol{x}):=V(\boldsymbol{x} / \delta) .
$$


Since $V \in D\left(\mathcal{B}_{\beta}\right)$, there holds $\mathrm{R}^{\delta} f \in \mathrm{H}^{2}\left(\Omega_{ \pm}^{\delta}\right)$. Moreover, we have $\left[\sigma \partial_{\boldsymbol{n}} V\right]_{\Gamma}=0$ so that $\mathrm{R}^{\delta} f=\hat{u}^{\delta}$ belongs to $D\left(\mathrm{~A}^{\delta}\right)$. Now observe that, since $r \leq r+\delta \leq 2 r$ on $\operatorname{supp}\left(\chi_{\delta}\right)$, there exists a constant $C>0$ independent of $\delta$ such that $\left\|\chi_{\delta} v\right\|_{\mathrm{V}_{\beta, \delta}^{2}(\Omega)} \leq C\left\|\chi_{\delta} v\right\|_{\mathrm{V}_{\beta}^{2}(\Omega)}$. Hence, using (23)-(25) with $\varepsilon=0$ we can check (work as in [30, Lem 2]) that

$$
\left\|\mathrm{R}^{\delta} f\right\|_{\mathrm{V}_{\beta, \delta}^{2}\left(\Omega \backslash \Gamma^{\delta}\right)}=\left\|\hat{u}^{\delta}\right\|_{\mathrm{V}_{\beta, \delta}^{2}\left(\Omega_{-}^{\delta}\right)}+\left\|\hat{u}^{\delta}\right\|_{\mathrm{V}_{\beta, \delta}^{2}\left(\Omega_{+}^{\delta}\right)} \leq C\|f\|_{\mathrm{V}_{\beta, \delta}^{0}(\Omega)} .
$$

We wish to prove that $\mathrm{R}^{\delta}$ is an approximate inverse of $\mathrm{A}^{\delta}$. Practically, we are going to show that $\hat{u}^{\delta}$ satisfies $\operatorname{div}\left(\sigma^{\delta} \nabla \hat{u}^{\delta}\right)=f^{\delta}$ for some source term $f^{\delta}$ close to $f$. A direct computation yields

$$
\begin{aligned}
-\operatorname{div}\left(\sigma^{\delta} \nabla \hat{u}^{\delta}\right) & =-\left[\operatorname{div}\left(\sigma^{\delta} \nabla \cdot\right), \chi_{\delta}\right] v-\left[\operatorname{div}\left(\sigma^{\delta} \nabla \cdot\right), \psi\right] V_{\delta}-\chi_{\delta} \operatorname{div}\left(\sigma^{\delta} \nabla v^{\delta}\right)-\psi \operatorname{div}\left(\sigma^{\delta} \nabla V_{\delta}\right) \\
& =-\left[\operatorname{div}\left(\sigma^{\delta} \nabla \cdot\right), \chi_{\delta}\right] v-\left[\operatorname{div}\left(\sigma^{\delta} \nabla \cdot\right), \psi\right] V_{\delta}+\chi_{\delta} \chi_{\sqrt{\delta}} f+\psi \psi_{\sqrt{\delta}} f \\
& =-\left[\operatorname{div}\left(\sigma^{\delta} \nabla \cdot\right), \chi_{\delta}\right] v-\left[\operatorname{div}\left(\sigma^{\delta} \nabla \cdot\right), \psi\right] V_{\delta}+f .
\end{aligned}
$$

In the above equalities, the commutator $[A, B]$ is defined by $[A, B]=A B-B A$. Since we have $\delta \leq r \leq 2 \delta$ on the support of $\left[\operatorname{div}\left(\sigma^{\delta} \nabla \cdot\right), \chi_{\delta}\right]$ and $1 \leq r \leq 2$ on the support of $\left[\operatorname{div}\left(\sigma^{\delta} \nabla \cdot\right)\right.$, $\left.\psi\right]$, we find that $\operatorname{div}\left(\sigma^{\delta} \nabla \hat{u}^{\delta}\right) \in \mathrm{V}_{\beta, \delta}^{0}(\Omega)$. Now, introduce the operator $\mathrm{K}^{\delta}: \mathrm{V}_{\beta, \delta}^{0}(\Omega) \rightarrow \mathrm{V}_{\beta, \delta}^{0}(\Omega)$ such that for all $f \in \mathrm{V}_{\beta, \delta}^{0}(\Omega), \mathrm{K}^{\delta} f$ denotes the function verifying

$$
-\operatorname{div}\left(\sigma^{\delta} \nabla \hat{u}^{\delta}\right)=f+\mathrm{K}^{\delta} f .
$$

From (28), we know that $\mathrm{K}^{\delta} f=-\left[\operatorname{div}\left(\sigma^{\delta} \nabla \cdot\right), \chi_{\delta}\right] v-\left[\operatorname{div}\left(\sigma^{\delta} \nabla \cdot\right), \psi\right] V_{\delta}$. Let us evaluate the norm of $\mathrm{K}^{\delta}$. First, remark that

$$
\left|\nabla \chi_{\delta}\right| \leq C \delta^{-1} \quad \text { and } \quad\left|\Delta \chi_{\delta}\right| \leq C \delta^{-2} .
$$

Defining, for $t>0$, the set $\mathbb{Q}^{t}:=\left\{\boldsymbol{x} \in \mathbb{R}^{3}|t<| \boldsymbol{x} \mid<2 t\right\}$, we can write

$$
\begin{aligned}
\left\|\left[\operatorname{div}\left(\sigma^{\delta} \nabla \cdot\right), \chi_{\delta}\right] v\right\|_{\mathrm{V}_{\beta, \delta}^{0}(\Omega)} & \leq\left\|(r+\delta)^{\beta} \sigma_{+} \nabla \chi_{\delta} \cdot \nabla v\right\|_{\Omega}+\left\|(r+\delta)^{\beta} \sigma_{+} \Delta \chi_{\delta} v\right\|_{\Omega} \\
& \leq C\left(\delta^{-1}\left\|(r+\delta)^{\beta} \nabla v\right\|_{\mathbb{Q}^{\delta}}+\delta^{-2}\left\|(r+\delta)^{\beta} v\right\|_{\mathbb{Q}^{\delta}}\right) \\
& \leq C \delta^{\varepsilon}\left(\left\|r^{\beta-\varepsilon-1} \nabla v\right\|_{\mathbb{Q}^{\delta}}+\left\|r^{\beta-\varepsilon-2} v\right\|_{\mathbb{Q}^{\delta}}\right) \\
& \leq C \delta^{\varepsilon}\|v\|_{\mathrm{V}_{\beta-\varepsilon}^{2}(\Omega)} \leq C \delta^{\varepsilon / 2}\|f\|_{\mathrm{V}_{\beta, \delta}^{0}} .
\end{aligned}
$$

In (30), the last inequality comes from (25), (23). Proceeding similarly, we find $\left\|\left[\operatorname{div}\left(\sigma^{\delta} \nabla \cdot\right), \psi\right] V_{\delta}\right\|_{\mathrm{V}_{\beta, \delta}^{0}(\Omega)} \leq$ $C \delta^{\varepsilon / 2}\|f\|_{\mathrm{V}_{\beta, \delta}^{0}}$. Therefore, for all $f \in \mathrm{V}_{\beta, \delta}^{0}(\Omega)$, there holds $\left\|\mathrm{K}^{\delta} f\right\|_{\mathrm{V}_{\beta, \delta}^{0}(\Omega)} \leq C \delta^{\varepsilon / 2}\|f\|_{\mathrm{V}_{\beta, \delta}^{0}(\Omega)}$. This proves that the norm of $\mathrm{K}^{\delta}: \mathrm{V}_{\beta, \delta}^{0}(\Omega) \rightarrow \mathrm{V}_{\beta, \delta}^{0}(\Omega)$ tends to zero when $\delta \rightarrow 0$. As a consequence, $\mathrm{Id}+\mathrm{K}^{\delta}$ is invertible for $\delta$ small enough.

From this discussion, since $\mathrm{A}^{\delta} \cdot \mathrm{R}^{\delta} f=\left(\mathrm{Id}+\mathrm{K}^{\delta}\right) f$, we conclude that the operator $\mathrm{A}^{\delta}$ admits $\mathrm{R}^{\delta}$. $\left(\mathrm{Id}+\mathrm{K}^{\delta}\right)^{-1}$ as a continuous inverse for $\delta$ small enough. This proves that there exists $\delta_{0}>0$ such that $\mathrm{A}^{\delta}$ is an isomorphism for all $\delta \in\left(0 ; \delta_{0}\right]$. Formula (26) implies that if $v \in D\left(\mathrm{~A}^{\delta}\right)$ verifies $\mathrm{A}^{\delta} v=f$ and if $\mathrm{A}^{\delta}$ is invertible, then we have $v=\mathrm{R}^{\delta} \cdot\left(\mathrm{Id}+\mathrm{K}^{\delta}\right)^{-1} f \in \mathrm{H}^{2}\left(\Omega_{ \pm}^{\delta}\right)$. Finally, we obtain (22) from estimates (27) and (30) which provide respectively uniform bounds of $\mathrm{R}^{\delta}$ and $\left(\mathrm{Id}+\mathrm{K}^{\delta}\right)^{-1}$.

\subsection{Asymptotic expansion for the source term problem}

Now that we know that problem (20) is uniquely solvable for $\delta$ small enough, we can think of providing an asymptotic expansion of its solution $u^{\delta}$ as $\delta$ goes to zero. This is precisely the goal of the present section. When $\delta \rightarrow 0$, as previously observed, the inclusion of negative material disappears. This leads us naturally to consider the following problem

$$
\mid \begin{aligned}
& \text { Find } v \in \mathrm{H}_{0}^{1}(\Omega) \text { such that } \\
& -\sigma_{+} \Delta v=f \quad \text { in } \Omega .
\end{aligned}
$$


Here, $f \in \mathrm{L}^{2}(\Omega)$ is the same source term as the one of (20). We have $\mathrm{L}^{2}(\Omega) \subset \mathrm{V}_{\beta}^{0}(\Omega)$ for all $\beta \in$ $(1 / 2 ; 3 / 2)$. Therefore, Proposition 2.1 ensures that Problem (31) has a unique solution $v=\left(\mathrm{A}^{0}\right)^{-1} f$ satisfying, for all $\beta \in(1 / 2 ; 3 / 2), v \in \mathrm{V}_{\beta}^{2}(\Omega)$. In addition, since $f \in \mathrm{L}^{2}(\Omega)$, it is known (see [29, Chap 1]) that $v-v(0)$ belongs to $\mathrm{V}_{0}^{2}(\Omega)$ with the estimate

$$
|v(0)|+\|v-v(0)\|_{\mathrm{V}_{0}^{2}(\Omega)} \leq C\|f\|_{\Omega} .
$$

In next proposition, we prove that for $\delta$ small enough, $v$ is a good approximation of $u^{\delta}$, the unique solution to Problem (20). To proceed, from $v$, we define another function $\mathcal{R}^{\delta} f$ by the formula

$$
\mathcal{R}^{\delta} f:=\chi_{\delta} v+\psi_{\delta} v(0),
$$

where $\chi_{\delta}, \psi_{\delta}$ were introduced in (5). The operator $\mathcal{R}^{\delta}$ is clearly linear, and estimate (32) indicates that it maps continuously $\mathrm{L}^{2}(\Omega)$ into $\mathrm{H}^{2}\left(\Omega \backslash \Gamma^{\delta}\right)$. Observe in addition that, since $\mathcal{R}^{\delta} v$ is constant in a neighbourhood of $\Omega_{-}^{\delta}$, we actually have $\mathcal{R}^{\delta} f \in D\left(\mathrm{~A}^{\delta}\right)$. The following result is obtained by observing that $\mathcal{R}^{\delta}$ approximates both $\left(\mathrm{A}^{\delta}\right)^{-1}$ and $\left(\mathrm{A}^{0}\right)^{-1}$.

Proposition 3.2. Assume that $\kappa_{\sigma} \neq-1$ and that Assumption 1 holds. Then for any $\beta \in(1 / 2,3 / 2)$, there exist constants $C_{\beta}, \delta_{0}>0$ independent of $\delta$ such that

$$
\sup _{f \in \mathrm{L}^{2}(\Omega) \backslash\{0\}} \frac{\left\|\left(\mathrm{A}^{0}\right)^{-1} f-\left(\mathrm{A}^{\delta}\right)^{-1} f\right\|_{\mathrm{V}_{\beta, \delta}^{2}\left(\Omega \backslash \Gamma^{\delta}\right)}}{\|f\|_{\Omega}} \leq C_{\beta} \delta^{\beta}, \quad \forall \delta \in\left(0 ; \delta_{0}\right] .
$$

Proof. Take any $f \in \mathrm{L}^{2}(\Omega)$ and for $\delta$ small enough, set $u^{\delta}:=\left(\mathrm{A}^{\delta}\right)^{-1} f, \hat{u}^{\delta}:=\mathcal{R}^{\delta} f, \tilde{v}:=v-v(0)$ where $v$ is the solution to (31). Since $1=\chi_{\delta}+\psi_{\delta}$, we have $\nabla \psi_{\delta}=-\nabla \chi_{\delta}$ and $\Delta \psi_{\delta}=-\Delta \chi_{\delta}$. This implies

$$
\begin{aligned}
-\operatorname{div}\left(\sigma^{\delta} \nabla\left(u^{\delta}-\hat{u}^{\delta}\right)\right) & =f+\operatorname{div}\left(\sigma^{\delta} \nabla \hat{u}^{\delta}\right) \\
& =f+\sigma_{+} \chi_{\delta} \Delta v+2 \sigma_{+} \nabla \chi_{\delta} \cdot \nabla \tilde{v}+\sigma_{+} \tilde{v} \Delta_{\delta} \\
& =f\left(1-\chi_{\delta}\right)+2 \sigma_{+} \nabla \chi_{\delta} \cdot \nabla \tilde{v}+\sigma_{+} \tilde{v} \Delta \chi_{\delta}:=\hat{f}^{\delta} .
\end{aligned}
$$

What precedes can be rewritten $\left(\mathrm{Id}-\mathrm{A}^{\delta} \cdot \mathcal{R}^{\delta}\right) f=\hat{f}^{\delta}$. Using (29), we find

$$
\begin{aligned}
\left\|\hat{f}^{\delta}\right\|_{\mathrm{V}_{\beta, \delta}^{0}(\Omega)} & \leq\left\|f\left(1-\chi_{\delta}\right)\right\|_{\mathrm{V}_{\beta, \delta}^{0}(\Omega)}+\left\|2 \sigma_{+} \nabla \chi_{\delta} \cdot \nabla \tilde{v}\right\|_{\mathrm{V}_{\beta, \delta}^{0}(\Omega)}+\left\|\sigma_{+} \tilde{v} \Delta \chi_{\delta}\right\|_{\mathrm{V}_{\beta, \delta}^{0}(\Omega)} \\
& \leq C\left(\left\|(r+\delta)^{\beta} f\right\|_{\mathrm{B}(O, 2 \delta)}+\delta^{-1}\left\|(r+\delta)^{\beta} \nabla \tilde{v}\right\|_{\mathbb{Q}^{\delta}}+\delta^{-2}\left\|(r+\delta)^{\beta} \tilde{v}\right\|_{\mathbb{Q}^{\delta}}\right) \\
& \leq C\left(\delta^{\beta}\|f\|_{\Omega}+\delta^{\beta}\|\tilde{v}\|_{\mathrm{V}_{0}^{2}(\Omega)} \leq C C \delta^{\beta}\|f\|_{\Omega} .\right.
\end{aligned}
$$

To obtain the last line of the above inequalities, we have used (32). This proves that $\|\left(\mathrm{Id}-\mathrm{A}^{\delta}\right.$. $\left.\mathcal{R}^{\delta}\right) f\left\|_{\mathrm{V}_{\beta, \delta}^{0}(\Omega)} \leq C \delta^{\beta}\right\| f \|_{\Omega}$ for all $f \in \mathrm{L}^{2}(\Omega)$. Now, observe that $\mathrm{A}^{\delta} \cdot \mathcal{R}^{\delta} f=\mathrm{A}^{0} \cdot \mathcal{R}^{\delta} f$. Therefore, we also have $\left\|\left(\mathrm{Id}-\mathrm{A}^{0} \cdot \mathcal{R}^{\delta}\right) f\right\|_{\mathrm{V}_{\beta, \delta}^{0}(\Omega)} \leq C \delta^{\beta}\|f\|_{\Omega}$ for all $f \in \mathrm{L}^{2}(\Omega)$. From Proposition 3.1 and Lemma 3.1 hereafter, we deduce

$$
\begin{aligned}
& \left\|\left(\mathrm{A}^{0}\right)^{-1} f-\left(\mathrm{A}^{\delta}\right)^{-1} f\right\|_{\mathrm{V}_{\beta, \delta}^{2}\left(\Omega \backslash \Gamma^{\delta}\right)} \\
\leq & \left\|\left(\mathrm{A}^{0}\right)^{-1} f-\mathcal{R}^{\delta} f\right\|_{\mathrm{V}_{\beta, \delta}^{2}\left(\Omega \backslash \Gamma^{\delta}\right)}+\left\|\left(\mathrm{A}^{\delta}\right)^{-1} f-\mathcal{R}^{\delta} f\right\|_{\mathrm{V}_{\beta, \delta}^{2}\left(\Omega \backslash \Gamma^{\delta}\right)} \\
\leq & C\left\|f-\mathrm{A}^{0} \cdot \mathcal{R}^{\delta} f\right\|_{\mathrm{V}_{\beta, \delta}^{0}(\Omega)}+C\left\|f-\mathrm{A}^{\delta} \cdot \mathcal{R}^{\delta} f\right\|_{\mathrm{V}_{\beta, \delta}^{0}(\Omega)} \leq C \delta^{\beta}\|f\|_{\Omega} .
\end{aligned}
$$

In (35), $C>0$ is a constant, which can change from one line to another, independent of $\delta, f$. With (35), we finally obtain (34).

The following lemma is a technical result needed in the proof of Proposition 3.2. It claims that the stability property $(22)$ for $\left(\mathrm{A}^{\delta}\right)^{-1}$ is also satisfied by $\left(\mathrm{A}^{0}\right)^{-1}$.

Lemma 3.1. For any $\beta \in(1 / 2,3 / 2)$, there exist constants $C_{\beta}, \delta_{0}>0$ independent of $\delta$ such that

$$
C_{\beta} \leq \inf _{v \in D\left(\mathrm{~A}^{0}\right) \backslash\{0\}} \frac{\left\|\mathrm{A}^{0} v\right\|_{\mathrm{V}_{\beta, \delta}^{0}(\Omega)}}{\|v\|_{\mathrm{V}_{\beta, \delta}^{2}\left(\Omega \backslash \Gamma^{\delta}\right)}}, \quad \forall \delta \in\left(0 ; \delta_{0}\right] .
$$


Proof. Decompose any element $v \in D\left(\mathrm{~A}^{0}\right)$ as $v(\boldsymbol{x})=\chi_{\delta}(\boldsymbol{x}) v(\boldsymbol{x})+V_{\delta}(\boldsymbol{x} / \delta)$ where $V_{\delta}(\boldsymbol{x} / \delta)=\psi_{\delta}(\boldsymbol{x}) v(\boldsymbol{x})$. We have $\operatorname{supp}\left(\chi_{\delta} v\right) \subset \Omega \backslash \mathrm{B}(O, 2 \delta)$, and $\operatorname{supp}\left(V_{\delta}\right) \subset \overline{\mathrm{B}}(O, 2)$. Observe that $D\left(\mathrm{~A}^{0}\right) \subset \mathrm{V}_{\beta}^{2}(\Omega)$ so that, according to Proposition 2.1, there holds $\|v\|_{\mathrm{V}_{\beta}^{2}(\Omega)} \leq C\|\Delta v\|_{\mathrm{V}_{\beta}^{0}(\Omega)}$. Here and in the sequel, $C>0$ denotes a constant independent of $\delta$ which can change from one line to another. As a consequence, we can write

$$
\begin{aligned}
\left\|\chi_{\delta} v\right\|_{\mathrm{V}_{\beta, \delta}^{2}(\Omega)} & \leq C\|v\|_{\mathrm{V}_{\beta, \delta}^{2}(\Omega \backslash \overline{\mathrm{B}(O, 2 \delta)})} \leq C\|v\|_{\mathrm{V}_{\beta}^{2}(\Omega \backslash \overline{\mathrm{B}(O, 2 \delta)})} \\
& \leq C\|v\|_{\mathrm{V}_{\beta}^{2}(\Omega)} \leq C\left\|\mathcal{A}_{\beta} v\right\|_{\mathrm{V}_{\beta}^{0}(\Omega)} \leq C\left\|\mathrm{~A}^{0} v\right\|_{\mathrm{V}_{\beta, \delta}^{0}(\Omega)} .
\end{aligned}
$$

In (36), we used that $r \leq r+\delta \leq 2 r$ in $\Omega \backslash \overline{\mathrm{B}(O, 2 \delta)}$ and that $\|v\|_{\mathrm{V}_{\beta}^{0}(\Omega)} \leq\|v\|_{\mathrm{V}_{\beta, \delta}^{0}(\Omega)}$ since $\beta \geq 0$. For $|\boldsymbol{\xi}| \geq 2$, there holds $V_{\delta}(\boldsymbol{\xi})=0$. Therefore, the usual elliptic a priori estimates give $\left\|V_{\delta}\right\|_{\mathcal{V}_{\beta}^{2}\left(\mathbb{R}^{3}\right)} \leq$ $C\left\|\Delta V_{\delta}\right\|_{\mathcal{V}_{\beta}^{0}\left(\mathbb{R}^{3}\right)}$. Making the change of variables $\boldsymbol{\xi}=\boldsymbol{x} / \delta$, we deduce

$$
\begin{aligned}
\left\|\psi_{\delta} v\right\|_{\mathrm{V}_{\beta, \delta}^{2}(\Omega)} & \leq C\left\|\Delta\left(\psi_{\delta} v\right)\right\|_{\mathrm{V}_{\beta, \delta}^{0}(\Omega)} \\
& \leq C\left\|\psi_{\delta} \Delta v\right\|_{\mathrm{V}_{\beta, \delta}^{0}(\Omega)}+C \delta^{-1}\|\nabla v\|_{\mathrm{V}_{\beta, \delta}^{0}\left(\mathbb{Q}^{\delta}\right)}+C \delta^{-2}\|v\|_{\mathrm{V}_{\beta, \delta}^{0}\left(\mathbb{Q}^{\delta}\right)} \\
& \leq C\left\|\mathrm{~A}^{0} v\right\|_{\mathrm{V}_{\beta, \delta}^{0}(\Omega)}+C \delta^{-1}\|\nabla v\|_{\mathrm{V}_{\beta}^{0}\left(\mathbb{Q}^{\delta}\right)}+C \delta^{-2}\|v\|_{\mathrm{V}_{\beta}^{0}\left(\mathbb{Q}^{\delta}\right)} \\
& \leq C\left\|\mathrm{~A}^{0} v\right\|_{\mathrm{V}_{\beta, \delta}^{0}(\Omega)}+C\|v\|_{\mathrm{V}_{\beta}^{2}(\Omega)} \\
& \leq C\left\|\mathrm{~A}^{0} v\right\|_{\mathrm{V}_{\beta, \delta}^{0}(\Omega)}+C\left\|\mathcal{A}_{\beta} v\right\|_{\mathrm{V}_{\beta}^{0}(\Omega)} \leq C\left\|\mathrm{~A}^{0} v\right\|_{\mathrm{V}_{\beta, \delta}^{0}(\Omega)} .
\end{aligned}
$$

There only remains to gather (36) and (37) that hold for any element $v \in D\left(\mathrm{~A}^{0}\right)$ and any $\delta \in\left(0 ; \delta_{0}\right]$. This leads to the conclusion of the proof.

Error estimate (34) can also be formulated in $\mathrm{L}^{2}$-norm. Indeed, observe that there exists a constant independent of $\delta$ such that $1 \leq C /(|\boldsymbol{x}|+\delta)$ for all $\boldsymbol{x} \in \Omega$. This implies $\|v\|_{\Omega} \leq C^{2-\beta}\|v\|_{\mathrm{V}_{\beta-2, \delta}^{0}(\Omega)} \leq$ $C^{2-\beta}\|v\|_{\mathrm{V}_{\beta, \delta}^{2}\left(\Omega \backslash \Gamma^{\delta}\right)}$ for all $v \in \mathrm{H}^{2}\left(\Omega \backslash \Gamma^{\delta}\right)$. From Proposition 3.2, we deduce the following result.

Corollary 3.1. Assume that $\kappa_{\sigma} \neq-1$ and that Assumption 1 holds. Then for any $\varepsilon \in(0 ; 1)$, there exist constants $C_{\varepsilon}, \delta_{0}>0$ independent of $\delta$ such that

$$
\sup _{f \in \mathrm{L}^{2}(\Omega) \backslash\{0\}} \frac{\left\|\left(\mathrm{A}^{0}\right)^{-1} f-\left(\mathrm{A}^{\delta}\right)^{-1} f\right\|_{\Omega}}{\|f\|_{\Omega}} \leq C_{\varepsilon} \delta^{3 / 2-\varepsilon}, \quad \forall \delta \in\left(0 ; \delta_{0}\right] .
$$

Remark 3.1. Notice that Proposition 3.2 and Corollary 3.1 prove that the function $v$ defined in (31) is a good approximation of $u^{\delta}$, the unique solution to Problem (20). Indeed, from (34) with $\beta=1$ and from (38), we can write, for all $\varepsilon \in(0 ; 1)$ and for $\delta$ small enough,

$$
\left\|u^{\delta}-v\right\|_{\mathrm{H}_{0}^{1}(\Omega)} \leq C \delta\|f\|_{\Omega} \quad \text { and } \quad\left\|u^{\delta}-v\right\|_{\Omega} \leq C_{\varepsilon} \delta^{3 / 2-\varepsilon}\|f\|_{\Omega} .
$$

This ends the asymptotic analysis of the source term problem. In the sequel, we turn back to the initial spectral problem. To make the transition, observe that the result of Corollary 3.1 can be rephrased as $\left(\mathrm{A}^{\delta}\right)^{-1}=\left(\mathrm{A}^{0}\right)^{-1}+O\left(\delta^{3 / 2-\varepsilon}\right)$ considering $\left(\mathrm{A}^{\delta}\right)^{-1},\left(\mathrm{~A}^{0}\right)^{-1}$ as operators mapping $\mathrm{L}^{2}(\Omega)$ to $\mathrm{L}^{2}(\Omega)$. Since these two operators are self-adjoint, a direct application of Theorem 4.10 of Chapter $\mathrm{V}$ of [20] yields that the spectra of $\left(\mathrm{A}^{\delta}\right)^{-1}$ and $\left(\mathrm{A}^{0}\right)^{-1}$ are closed to each other.

Proposition 3.3. Assume that $\kappa_{\sigma} \neq-1$ and that Assumption 1 holds. Then for any $\varepsilon \in(0 ; 1)$, there exist constants $C_{\varepsilon}, \delta_{0}>0$ independent of $\delta$ such that

$$
\sup _{\mu \in \mathfrak{S}\left(\mathrm{A}^{0}\right)} \inf _{\lambda \in \mathfrak{S}\left(\mathrm{A}^{\delta}\right)}\left|\frac{1}{\lambda}-\frac{1}{\mu}\right|+\sup _{\lambda \in \mathfrak{S}\left(\mathrm{A}^{\delta}\right)} \inf _{\mu \in \mathfrak{S}\left(\mathrm{A}^{0}\right)}\left|\frac{1}{\lambda}-\frac{1}{\mu}\right| \leq C_{\varepsilon} \delta^{3 / 2-\varepsilon}, \quad \forall \delta \in\left(0 ; \delta_{0}\right] .
$$

Remark 3.2. The use of [20, Thm 4.10, Chap V] (or Lemma 6.1 in Appendix) is crucial here in order to convert consistency estimates into spectral estimates. This result requires that the operator under consideration be at least normal. When the latter assumption is not satisfied, probably new significant difficulties arise. 


\section{Study of the positive spectrum}

In this section, we exploit Proposition 3.3 to prove our main result concerning the positive spectrum of $\mathrm{A}^{\delta}$, namely, it converges to the spectrum of $\mathrm{A}^{0}$.

Theorem 4.1. Assume that $\kappa_{\sigma} \neq-1$ and that Assumption 1 holds. Let $n \in \mathbb{N}^{*}$ be a fixed number and let $\lambda_{n}^{\delta}>0$ (resp. $\left.\mu_{n}>0\right)$ refer to the corresponding eigenvalue of the operator $\mathrm{A}^{\delta}$ (resp. $\left.\mathrm{A}^{0}\right)$. Then there exist constants $C_{\varepsilon}, \delta_{0}>0$, depending on $n, \varepsilon$ but independent of $\delta$, such that

$$
\left|\mu_{n}-\lambda_{n}^{\delta}\right| \leq C_{\varepsilon} \delta^{3 / 2-\varepsilon}, \quad \forall \delta \in\left(0 ; \delta_{0}\right] .
$$

Proof. Pick $\varepsilon \in(0 ; 1)$. Let $\mu_{n}, n \in \mathbb{N}^{*}$, be an eigenvalue of multiplicity $\varkappa \geq 1$ of the far field operator $\mathrm{A}^{0}$. To set our ideas and without loss of generality, we assume that there holds

$$
\mu_{n-1}<\mu_{n}=\cdots=\mu_{n+\varkappa-1}<\mu_{n+\varkappa}
$$

if $n>1$. Estimate (34) establishes strong convergence of $\left(\mathrm{A}^{\delta}\right)^{-1}$ toward $\left(\mathrm{A}^{0}\right)^{-1}$. Therefore, we can apply Theorem 3.16 of Chapter IV of [20] which ensures that for $C>0$ large enough, for all $\delta \in\left(0 ; \delta_{0}\right]$ and all $i \in\{1, \ldots, n\}$, the total multiplicity of the spectrum of $\left(\mathrm{A}^{\delta}\right)^{-1}$ in $\mathcal{O}_{i}^{\delta}:=\left[\mu_{i}^{-1}-C \delta^{3 / 2-\varepsilon} ; \mu_{i}^{-1}+\right.$ $\left.C \delta^{3 / 2-\varepsilon}\right]$ is equal to $\varkappa_{i}$, the multiplicity of $\mu_{i}^{-1}$. We denote $\lambda_{k}^{\delta} \leq \cdots \leq \lambda_{k+\varkappa-1}^{\delta}$, for some $k \in \mathbb{N}^{*}$, the eigenvalues of $\mathrm{A}^{\delta}$ whose inverses are located in $\left[\mu_{n}^{-1}-C \delta^{3 / 2-\varepsilon} ; \mu_{n}^{-1}+C \delta^{3 / 2-\varepsilon}\right]$. Now, we wish to prove that for all $\delta \in\left(0 ; \delta_{0}\right]$, we have $k=n$. For all $i<n$ and $\delta \in\left(0 ; \delta_{0}\right]$, we just showed that there are exactly $\varkappa_{i}$ eigenvalues of $\left(\mathrm{A}^{\delta}\right)^{-1}$ in $\mathcal{O}_{i}^{\delta}$. As a consequence, the relation $k \geq n$ is valid. Now, assume that $k>n$ for some $\delta \in\left(0 ; \delta_{0}\right]$. In this case, because of the relation on the multiplicity, there exists some $j \in \mathbb{N}^{*}$ such that $1 / \lambda_{j}^{\delta} \notin \cup_{i=1}^{n-1} \mathcal{O}_{i}^{\delta}$. If the $C$ of the definition of the $\mathcal{O}_{i}^{\delta}$ is larger than the $C_{\varepsilon}$ of estimate (39), this is impossible. Therefore, we have $k=n$ and $\left|1 / \mu_{n}-1 / \lambda_{n}^{\delta}\right| \leq C \delta^{3 / 2-\varepsilon}$. To conclude, it remains to notice that the latter inequality is equivalent to (40) because $\mu_{n}>0$.

Remark 4.1. Observe that in (40), the dependence of $C_{\varepsilon}$ with respect to $n \in \mathbb{N}^{*}$ can be explicitly computed since there holds $C_{\varepsilon}=O\left(\mu_{n}^{2}\right)$ when $n \rightarrow+\infty$.

\section{$5 \quad$ Study of the negative spectrum}

The presence of negative eigenvalues in the spectrum of $\mathrm{A}^{\delta}$ is due to the small piece of negative material in the domain $\Omega$. We will see that the behaviour of negative eigenvalues is driven by the near field structure of the operator $\mathrm{A}^{\delta}$. More precisely, we will prove that the negative part of the spectrum of $\mathrm{A}^{\delta}$ is asymptotically equivalent (in a precise meaning that we shall provide later) to the negative part of the spectrum of $\delta^{-2} \mathrm{~B}^{\infty}$. We shall proceed in two steps (so called inverse and direct reductions) following and adapting the approach considered in [30].

Let us first introduce notation adapted to the study of the operator $\mathrm{A}^{\delta}$ close to the small inclusion. Throughout this section, we shall make intensive use of the fast variable $\boldsymbol{\xi}=\boldsymbol{x} / \delta$. In this rescaled variable, the domain $\Omega$ becomes the $\delta$-dependent domain $\Xi^{\delta}:=\left\{\boldsymbol{\xi} \in \mathbb{R}^{3} \mid \delta \boldsymbol{\xi} \in \Omega\right\}$ while the operator $\mathrm{A}^{\delta}$ is changed into the operator $\mathrm{B}^{\delta}: D\left(\mathrm{~B}^{\delta}\right) \rightarrow \mathrm{L}^{2}\left(\Xi^{\delta}\right)$ defined by

$$
\mid \begin{aligned}
& \mathrm{B}^{\delta} w^{\delta}=-\operatorname{div}\left(\sigma^{\infty} \nabla w^{\delta}\right) \\
& D\left(\mathrm{~B}^{\delta}\right):=\left\{w^{\delta} \in \mathrm{H}_{0}^{1}\left(\Xi^{\delta}\right) \mid \operatorname{div}\left(\sigma^{\infty} \nabla w^{\delta}\right) \in \mathrm{L}^{2}\left(\Xi^{\delta}\right)\right\} .
\end{aligned}
$$

A simple calculus shows directly that $\mathfrak{S}\left(\mathrm{B}^{\delta}\right)=\left\{\delta^{2} \lambda \mid \lambda \in \mathfrak{S}\left(\mathrm{A}^{\delta}\right)\right\}$. Therefore, $\mathfrak{S}\left(\mathrm{B}^{\delta}\right)$ consists in the discrete set of ordered real values $\cdots \leq \delta^{2} \lambda_{-2}^{\delta} \leq \delta^{2} \lambda_{-1}^{\delta}<0 \leq \delta^{2} \lambda_{1}^{\delta} \leq \delta^{2} \lambda_{2}^{\delta} \leq \ldots$. Here we will focus on the negative part of this set, as we want to show that $\mathfrak{S}\left(\mathrm{B}^{\delta}\right) \backslash \overline{\mathbb{R}_{+}}$and $\mathfrak{S}\left(\mathrm{B}^{\infty}\right) \backslash \overline{\mathbb{R}_{+}}$get close to each other as $\delta \rightarrow 0$. From the study of $\S 3.2$, we can already state the following preliminary result concerning the negative spectrum of $\mathrm{A}^{\delta}$. 
Lemma 5.1. Assume that $\kappa_{\sigma} \neq-1$ and that Assumption 1 holds. Then for any $\varepsilon \in(0 ; 1)$, there exist constants $C_{\varepsilon}, \delta_{0}>0$ independent of $\delta$ such that

$$
\mathfrak{S}\left(\mathrm{A}^{\delta}\right) \cap\left(-C_{\varepsilon} \delta^{-3 / 2+\varepsilon} ; 0\right)=\emptyset, \quad \forall \delta \in\left(0 ; \delta_{0}\right] .
$$

Proof. Proposition 3.3 ensures that

$$
\sup _{\lambda \in \mathfrak{S}\left(\mathrm{A}^{\delta}\right)} \inf _{\mu \in \mathfrak{S}\left(\mathrm{A}^{0}\right)}\left|\frac{1}{\lambda}-\frac{1}{\mu}\right| \leq C_{\varepsilon} \delta^{3 / 2-\varepsilon}, \quad \forall \delta \in\left(0 ; \delta_{0}\right] .
$$

But we know that the spectrum of $\mathrm{A}^{0}$ is strictly positive. Therefore, for all $\delta \in\left(0 ; \delta_{0}\right]$, we have $\sup _{\lambda \in \mathfrak{S}\left(\mathrm{A}^{\delta}\right) \backslash \overline{\mathbb{R}_{+}}}|\lambda|^{-1} \leq C_{\varepsilon} \delta^{3 / 2-\varepsilon}$. This implies (41).

\subsection{Inverse reduction}

In this section, we establish that if $\mu_{-n}$ is an eigenvalue of $\mathrm{B}^{\infty}$, then close to $\delta^{-2} \mu_{-n}$ there is an element of the negative part of the spectrum of $\mathrm{B}^{\delta}$. The method is classical: from eigenpairs of the limit operator $\mathrm{B}^{\infty}$, we construct approximation of eigenpairs of $\mathrm{B}^{\delta}$. Then, we use the well-known lemma on "near eigenvalues and eigenfunctions" (see [47]). Since we start from eigenpairs of the limit problem to build eigenpairs for the original problem, this approach is usually called inverse reduction. We will see that the exponential decay of the eigenfunctions of $\mathrm{B}^{\infty}$ established in $\S 2.2$ induces exponential convergence.

Lemma 5.2. Assume that $\kappa_{\sigma} \neq-1$. For any $\mu \in \mathfrak{S}\left(\mathrm{B}^{\infty}\right) \backslash \overline{\mathbb{R}_{+}}$, there exist $C, \delta_{0}>0$ independent of $\delta$ such that

$$
\inf _{w^{\delta} \in D\left(\mathrm{~B}^{\delta}\right) \backslash\{0\}} \frac{\left\|\mathrm{B}^{\delta} w^{\delta}-\mu w^{\delta}\right\|_{\Xi^{\delta}}}{\left\|w^{\delta}\right\|_{\Xi^{\delta}}} \leq C \delta \exp \left(-\frac{1}{2 \delta} \sqrt{\frac{|\mu|}{\sigma_{+}}}\right), \quad \forall \delta \in\left(0 ; \delta_{0}\right] .
$$

Proof. Pick an arbitrary $\mu \in \mathfrak{S}\left(\mathrm{B}^{\infty}\right) \backslash \overline{\mathbb{R}_{+}}$and consider a corresponding eigenfunction $w$ that satisfies $\|w\|_{\mathbb{R}^{3}}=1$. Set $w^{\delta} \in D\left(\mathrm{~B}^{\delta}\right)$ by $w^{\delta}=\psi^{\delta} w$, where $\psi^{\delta}$ is the function such that $\psi^{\delta}(\boldsymbol{\xi}):=\psi(\delta \boldsymbol{\xi})$ (see (4) for the defintion of $\psi$ ). Let us use $w^{\delta}$ to prove (42). We have $\operatorname{div}\left(\sigma^{\infty} \nabla w^{\delta}\right)=\psi^{\delta} \operatorname{div}\left(\sigma^{\infty} \nabla w\right)+$ $2 \sigma_{+} \nabla \psi^{\delta} \cdot \nabla w+\sigma_{+} w \Delta \psi^{\delta}$. Since $\nabla \psi^{\delta}, \Delta \psi^{\delta}$ vanish outside $\mathbb{Q}^{1 / \delta}=\left\{\boldsymbol{\xi} \in \mathbb{R}^{3}\left|\delta^{-1}<\right| \boldsymbol{\xi} \mid<2 \delta^{-1}\right\}$, applying Proposition 2.3, we see that for $\gamma=\sqrt{|\mu| / \sigma_{+}}$, we have

$$
\begin{aligned}
\left\|\mathrm{B}^{\delta} w^{\delta}-\mu w^{\delta}\right\|_{\Xi^{\delta}}^{2} & =\left\|2 \sigma_{+} \nabla \psi^{\delta} \cdot \nabla w+\sigma_{+} w \Delta \psi^{\delta}\right\|_{\Xi^{\delta}}^{2} \\
& \leq C\left(\delta^{2}\|\nabla w\|_{\mathbb{Q}^{1 / \delta}}^{2}+\delta^{4}\|w\|_{\mathbb{Q}^{1 / \delta}}^{2}\right) \\
& \leq C \delta^{2} \exp (-\gamma / \delta) \int_{\mathbb{R}^{3}}\left(|w|^{2}+|\nabla w|^{2}\right) \exp (\gamma|\boldsymbol{\xi}|) d \boldsymbol{\xi} .
\end{aligned}
$$

On the other hand, using the dominated convergence theorem, we see that $\left\|w^{\delta}\right\|_{\Xi^{\delta}}$ tends to $\|w\|_{\mathbb{R}^{3}}=1$ when $\delta \rightarrow 0$. As a consequence, we have $\left\|w^{\delta}\right\|_{\Xi^{\delta}} \geq c>0$ for $\delta$ small enough, so that $\| \mathrm{B}^{\delta} w^{\delta}-$ $\mu w^{\delta}\left\|_{\Xi^{\delta}} /\right\| w^{\delta} \|_{\Xi^{\delta}}=O(\delta \exp (-\gamma / 2 \delta))$.

To obtain the next proposition, we use Lemma 5.2 and we apply the classical result on "near eigenvalues and eigenfunctions" (see Lemma 6.1 in appendix) to $\mathrm{B}^{\delta}: D\left(\mathrm{~B}^{\delta}\right) \rightarrow \mathrm{L}^{2}\left(\Xi^{\delta}\right)$. Observe that the latter operator, which is self-adjoint, is indeed normal.

Proposition 5.1. Assume that $\kappa_{\sigma} \neq-1$. For any $\mu \in \mathfrak{S}\left(\mathrm{B}^{\infty}\right) \backslash \overline{\mathbb{R}_{+}}$, there exists $C, \gamma, \delta_{0}>0$ independent of $\delta$ such that

$$
\inf _{\lambda \in \mathfrak{S}\left(\mathrm{A}^{\delta}\right)}\left|\lambda-\delta^{-2} \mu\right| \leq C \exp (-\gamma / \delta), \quad \forall \delta \in\left(0 ; \delta_{0}\right] .
$$

With Lemma 5.1, we have seen that for all $\varepsilon>0$, the set $\mathfrak{S}\left(\mathrm{A}^{\delta}\right) \cap\left(-C_{\varepsilon} \delta^{-3 / 2+\varepsilon} ; 0\right)$ is empty for $\delta$ small enough. The next result indicates that, as $\delta \rightarrow 0$, the negative eigenvalues of $\mathrm{A}^{\delta}$ tend to $-\infty$ not too fast though. 
Corollary 5.1. Assume that $\kappa_{\sigma} \neq-1$. Let $n \in \mathbb{N}^{*}$ be a fixed number and let $\lambda_{-n}^{\delta}<0$ refer to the corresponding eigenvalue of the operator $\mathrm{A}^{\delta}$ as defined by Proposition 3. Then, we have $\lim \sup _{\delta \rightarrow 0} \delta^{2}\left|\lambda_{-n}^{\delta}\right|<+\infty$.

Proof. Assume that there exist $n \in \mathbb{N}^{*}$ and a sequence $\delta_{k} \rightarrow 0$ such that $\lim _{k \rightarrow+\infty} \delta_{k}^{2} \lambda_{-n}^{\delta_{k}}=-\infty$. Since we have $\lambda_{-m} \leq \lambda_{-n}$ for $m \geq n$, we conclude that $\lim _{k \rightarrow+\infty} \delta_{k}^{2} \lambda_{-m}^{\delta_{k}}=-\infty$ for all $m \geq n$. So only the sequences $\left(\delta_{k}^{2} \lambda_{-m}^{\delta_{k}}\right)_{k \geq 0}$ with $m \in\{1, \ldots, n-1\}$ may possibly remain bounded.

Now, consider $0>\mu^{\ell_{1}}>\cdots>\mu^{\ell_{n}} n$ distinct elements of $\mathfrak{S}\left(\mathrm{B}^{\infty}\right) \backslash \overline{\mathbb{R}_{+}}$. According to Proposition 5.1, there exist constants $C, \gamma, \delta_{0}>0$ independent of $\delta$ such that each interval $\left[\mu^{\ell_{m}}-C \delta^{2} \exp (-\gamma / \delta) ; \mu^{\ell_{m}}+\right.$ $\left.C \delta^{2} \exp (-\gamma / \delta)\right], m=1 \ldots n$, contains at least one element of the form $\delta^{2} \lambda_{j}^{\delta}$ for all $\delta \in\left(0 ; \delta_{0}\right]$. This imposes that we have $\lim \sup _{\delta \rightarrow 0} \delta^{2}\left|\lambda_{j}^{\delta}\right|<+\infty$ for at least $n$ distinct eigenvalues $\lambda_{j}^{\delta} \in \mathfrak{S}\left(\mathrm{A}^{\delta}\right)$. This is in contradiction with what precedes. Therefore, there holds $\lim \sup _{\delta \rightarrow 0} \delta^{2}\left|\lambda_{-n}^{\delta}\right|<+\infty$ for all $n \in \mathbb{N}^{*}$.

\subsection{Direct reduction}

From Proposition 5.1, we know that for all $n \in \mathbb{N}^{*}$, if $\mu_{-n}$ is an eigenvalue of $\mathrm{B}^{\infty}$, then close to $\mu_{-n}$ there is a negative eigenvalue of $\mathrm{B}^{\delta}$. In this section, we prove the converse assertion. We use the same technique as in the previous section: from eigenpairs of $\mathrm{B}^{\delta}$, we build approximations of eigenpairs of the limit operator $\mathrm{B}^{\infty}$. Then, we conclude thanks to the lemma on "near eigenvalues and eigenfunctions". This process is called the direct reduction because we start from eigenpairs of the original problem to construct eigenpairs of the limit problem. As is often the case, the direct reduction will be slightly more complicated than the inverse reduction. We start by a preliminary lemma where we show a localization effect for the eigenfunctions of $\mathrm{B}^{\delta}$ associated with the negative eigenvalues.

Lemma 5.3. Assume that $\kappa_{\sigma} \neq-1$ and that Assumption 1 holds. Let $n \in \mathbb{N}^{*}$ be a fixed number and let $w_{-n}^{\delta}$, such that $\left\|w_{-n}^{\delta}\right\|_{\Xi^{\delta}}=1$, refer to an eigenfunction of $\mathrm{B}^{\delta}$ corresponding to the negative eigenvalue $\delta^{2} \lambda_{-n}^{\delta}$. Then, we have

$$
\limsup _{\delta \rightarrow 0} \int_{\Xi^{\delta}}\left(\delta^{2}\left|\lambda_{-n}^{\delta}\right|\left|w_{-n}^{\delta}(\boldsymbol{\xi})\right|^{2}+\left|\nabla w_{-n}^{\delta}(\boldsymbol{\xi})\right|^{2}\right) \exp \left(|\boldsymbol{\xi}| \sqrt{\frac{\delta^{2}\left|\lambda_{-n}^{\delta}\right|}{\sigma_{+}}}\right) d \boldsymbol{\xi}<+\infty
$$

Proof. To simplify the notation, let us drop the index " ${ }_{-n}$ ". We adopt here the same approach as in Proposition 2.3. Let us introduce the weight function $\mathscr{W}^{\delta} \operatorname{such}$ that $\mathscr{W}^{\delta}(\boldsymbol{\xi}):=\exp \left(\gamma^{\delta}\right)$ for $|\boldsymbol{\xi}| \leq 1$ and $\mathscr{W}^{\delta}(\boldsymbol{\xi}):=\exp \left(\gamma^{\delta}|\boldsymbol{\xi}|\right)$ for $|\boldsymbol{\xi}| \geq 1$, with $\gamma^{\delta}=\left(\left|\delta^{2} \lambda^{\delta}\right| / \sigma_{+}\right)^{1 / 2} / 2$. Since $-\operatorname{div}\left(\sigma^{\infty} \nabla w^{\delta}\right)=\delta^{2} \lambda^{\delta} w^{\delta}$ in $\Xi^{\delta}$, a computation nearly identical to that of the proof of Proposition 2.3 shows that

$$
\delta^{2}\left|\lambda^{\delta}\right|\left\|\mathscr{W}^{\delta} w^{\delta}\right\|_{\Xi^{\delta}}^{2}+\sigma_{+}\left\|\mathscr{W}^{\delta} \nabla w^{\delta}\right\|_{\Xi^{\delta}}^{2} \leq 2 \exp \left(2 \gamma^{\delta}\right)\left(\sigma_{+}+\left|\sigma_{-}\right|\right)\left\|\nabla w^{\delta}\right\|_{\Xi_{-}}^{2} .
$$

From Corollary 5.1, we know that the map $\delta \mapsto \delta^{2}\left|\lambda^{\delta}\right|$ (and so $\delta \mapsto \gamma^{\delta}$ ) is bounded as $\delta \rightarrow 0$. In addition, applying (12) yields a constant $C>0$ independent of $\delta, \lambda^{\delta}$, such that $\left\|\nabla w^{\delta}\right\|_{\Xi_{-}} \leq$ $C\left(1+\delta^{2}\left|\lambda^{\delta}\right|\right)\left\|w^{\delta}\right\|_{\Xi_{-}}$. Since $\left\|w^{\delta}\right\|_{\Xi_{-}} \leq\left\|w^{\delta}\right\|_{\Xi^{\delta}}=1$, we deduce that the right-hand side of the estimate (45) remains bounded uniformly as $\delta \rightarrow 0$. This ends the proof.

Note that if $w_{-n}^{\delta}$ is an eigenfunction of $\mathrm{B}^{\delta}$ with $\left\|w_{-n}^{\delta}\right\|_{\Xi^{\delta}}=1$, then the function $v^{\delta}$ such that $v^{\delta}(\boldsymbol{x})=$ $\delta^{3 / 2} w_{-n}^{\delta}(\boldsymbol{x} / \delta)$ is an eigenfunction of $\mathrm{A}^{\delta}$ with $\left\|v^{\delta}\right\|_{\Xi^{\delta}}=1$. Therefore, the previous lemma shows that eigenfunctions of $\mathrm{A}^{\delta}$ associated with negative eigenvalues get more and more localized around the small negative inclusion as $\delta \rightarrow 0$. Now, we can construct quasi-eigenfunctions for $\mathrm{B}^{\infty}$ from eigenfunctions of the operator $\mathrm{B}^{\delta}$.

Lemma 5.4. Assume that $\kappa_{\sigma} \neq-1$ and that Assumption 1 holds. Let $n \in \mathbb{N}^{*}$ be a fixed number and let $\delta^{2} \lambda_{-n}^{\delta}<0$ refer to the corresponding eigenvalue of the operator $\mathrm{B}^{\delta}$. Then there exist constants $C, \gamma, \delta_{0}>0$, depending on $n$ but independent of $\delta$, such that

$$
\inf _{w \in D\left(\mathrm{~B}^{\infty}\right) \backslash\{0\}} \frac{\left\|\mathrm{B}^{\infty} w-\delta^{2} \lambda_{-n}^{\delta} w\right\|_{\mathbb{R}^{3}}}{\|w\|_{\mathbb{R}^{3}}} \leq C \delta \exp (-\gamma / \delta), \quad \forall \delta \in\left(0 ; \delta_{0}\right] .
$$


Proof. Once again, we omit the index " ${ }_{-n} n$ ". Let us consider an eigenfunction $w^{\delta}$ of $\mathrm{B}^{\delta}$ associated with the eigenvalue $\delta^{2} \lambda^{\delta}$. We assume that there holds $\left\|w^{\delta}\right\|_{\Xi^{\delta}}=1$. Set $w:=\psi^{\delta} w^{\delta}$ where $\psi^{\delta}$ is such that $\psi^{\delta}(\boldsymbol{\xi}):=\psi(\delta \boldsymbol{\xi})(\psi$ is the cut-off function defined in $(4))$. Let us show first that

$$
\lim _{\delta \rightarrow 0}\|w\|_{\mathbb{R}^{3}}=1 .
$$

Introducing $\chi^{\delta}=1-\psi^{\delta}$, we can write $\|w\|_{\mathbb{R}^{3}}=\left\|\psi^{\delta} w^{\delta}\right\|_{\mathbb{R}^{3}} \geq\left\|w^{\delta}\right\|_{\Xi^{\delta}}-\left\|\chi^{\delta} w^{\delta}\right\|_{\Xi^{\delta}}$. According to Lemma 5.1, there exists a constant $C>0$ independent of $\delta$ such that $\left|\lambda^{\delta}\right| \geq C \delta^{-1}$ so that $\delta^{2}\left|\lambda^{\delta}\right| \geq C \delta$ for $\delta$ small enough. In addition, observe that $|\boldsymbol{\xi}| \geq 1 / \delta$ for $\boldsymbol{\xi} \in \operatorname{supp}\left(\chi^{\delta}\right)$. As a consequence, applying Lemma 5.3 above, we can write

$$
\left\|\chi^{\delta} w^{\delta}\right\|_{\Xi^{\delta}}^{2} \leq \frac{\exp \left(-\sqrt{\left|\lambda^{\delta}\right| / \sigma_{+}}\right)}{\delta^{2}\left|\lambda^{\delta}\right|} \int_{\Xi^{\delta}} \delta^{2}\left|\lambda^{\delta}\right|\left|w^{\delta}(\boldsymbol{\xi})\right|^{2} \exp \left(|\boldsymbol{\xi}| \sqrt{\frac{\delta^{2}\left|\lambda^{\delta}\right|}{\sigma_{+}}}\right) d \boldsymbol{\xi} \leq C_{1} \delta^{-1} \exp \left(-C_{2} \delta^{-1 / 2}\right) .
$$

This implies $\lim _{\delta \rightarrow 0}\left\|\chi^{\delta} w^{\delta}\right\|_{\Xi^{\delta}}=0$ and establishes (47). Now, we estimate $\left\|\mathrm{B}^{\infty} w-\delta^{2} \lambda w\right\|_{\mathbb{R}^{3}}$. We compute $\operatorname{div}\left(\sigma^{\infty} \nabla w\right)=\psi^{\delta} \operatorname{div}\left(\sigma^{\infty} \nabla w^{\delta}\right)+2 \sigma_{+} \nabla \psi^{\delta} \cdot \nabla w^{\delta}+\sigma_{+} w^{\delta} \Delta \psi^{\delta}$. Observing that $\left|\nabla \psi^{\delta}\right| \leq$ $C \delta,\left|\Delta \psi^{\delta}\right| \leq C \delta^{2}$ are non null only on $\mathbb{Q}^{1 / \delta}=\left\{\boldsymbol{\xi} \in \mathbb{R}^{3}\left|\delta^{-1}<\right| \boldsymbol{\xi} \mid<2 \delta^{-2}\right\}$ and recalling that $\operatorname{div}\left(\sigma^{\infty} \nabla w^{\delta}\right)=\delta^{2} \lambda^{\delta} w^{\delta}$, we conclude that there exists a constant $C>0$ independent of $\delta$ such that

$$
\begin{aligned}
\left\|\mathrm{B}^{\infty} w-\delta^{2} \lambda^{\delta} w\right\|_{\mathbb{R}^{3}} & =\left\|2 \sigma_{+} \nabla \psi^{\delta} \cdot \nabla w^{\delta}+\sigma_{+} w^{\delta} \Delta \psi^{\delta}\right\|_{\Xi^{\delta}} \\
& \leq C\left(\delta\left\|\nabla w^{\delta}\right\|_{\mathbb{Q}^{1 / \delta}}+\delta^{2}\left\|w^{\delta}\right\|_{\mathbb{Q}^{1 / \delta}}\right)
\end{aligned}
$$

Using Lemma 5.3 and working as in the beginning of this proof, we can show that lim $\sup _{\delta \rightarrow 0}\left\|w^{\delta}\right\|_{\Xi^{\delta}}+$ $\left\|\nabla w^{\delta}\right\|_{\Xi^{\delta}}<+\infty$. This remark, combined with the above inequality shows that $\left\|\mathrm{B}^{\infty} w-\delta^{2} \lambda^{\delta} w\right\|_{\mathbb{R}^{3}}=$ $O(\delta)$. Since $\mathrm{B}^{\infty}$ is self-adjoint, we can apply the lemma on "near eigenvalues and eigenfunctions" (see Lemma 6.1 in appendix) to obtain

$$
\inf _{\mu \in \mathfrak{S}\left(\mathrm{B}^{\infty}\right)}\left|\mu-\delta^{2} \lambda^{\delta}\right|=O(\delta)
$$

We deduce in particular that there exists $\delta_{0}>0$ independent of $\delta$ such that

$$
\delta^{2}\left|\lambda^{\delta}\right| \geq\left|\mu_{-1}\right| / 2, \quad \forall \delta \in\left(0 ; \delta_{0}\right] .
$$

Now, let us come back to (48). Since $\delta^{-1} \leq|\boldsymbol{\xi}| \leq 2 \delta^{-1}$ on $\mathbb{Q}^{1 / \delta}$, making use of (50) combined with Lemma 5.3, we deduce that there exists a constant $C>0$ independent of $\delta$ such that $\| \mathrm{B}^{\infty} w^{\delta}-$ $\delta^{2} \lambda_{-n}^{\delta} w^{\delta} \|_{\mathbb{R}^{3}} \leq C \delta \exp \left(-\delta^{-1} \sqrt{\left|\mu_{-1}\right| /\left(2 \sigma_{+}\right)}\right)$. This, together with (47) leads to the conclusion of the proof.

Apply one last time Lemma 6.1 in appendix to obtain the

Proposition 5.2. Assume that $\kappa_{\sigma} \neq-1$ and that Assumption 1 holds. Let $n \in \mathbb{N}^{*}$ be a fixed number and let $\lambda_{-n}^{\delta}<0$ refer to the corresponding eigenvalue of the operator $\mathrm{A}^{\delta}$. Then there exist constants $C, \gamma, \delta_{0}>0$, depending on $n$ but independent of $\delta$, such that

$$
\inf _{\mu \in \mathfrak{S}\left(\mathrm{B}^{\infty}\right)}\left|\lambda_{-n}^{\delta}-\delta^{-2} \mu\right| \leq C \exp (-\gamma / \delta), \quad \forall \delta \in\left(0 ; \delta_{0}\right]
$$

\subsection{Conclusion}

In the next theorem, we state the main result of the paper concerning the negative spectrum of the original operator $\mathrm{A}^{\delta}$.

Theorem 5.1. Assume that $\kappa_{\sigma} \neq-1$ and that Assumption 1 holds. Let $n \in \mathbb{N}^{*}$ be a fixed number and let $\lambda_{-n}^{\delta}<0$ (resp. $\mu_{-n}<0$ ) refer to the corresponding eigenvalue of the operator $\mathrm{A}^{\delta}$ (resp. $\left.\mathrm{B}^{\infty}\right)$. Then there exist constants $C, \gamma, \delta_{0}>0$, depending on $n$ but independent of $\delta$, such that

$$
\left|\lambda_{-n}^{\delta}-\delta^{-2} \mu_{-n}\right| \leq C \exp (-\gamma / \delta), \quad \forall \delta \in\left(0 ; \delta_{0}\right]
$$


Proof. Pick some arbitrary $n \in \mathbb{N}^{*}$. Corollary 5.1 ensures that the map $\delta \mapsto \delta^{2} \lambda_{-n}^{\delta}$ remains bounded on $\left(0 ; \delta_{0}\right]$ for some $\delta_{0}>0$. From Proposition 5.2, we deduce that $\delta \mapsto \delta^{2} \lambda_{-n}^{\delta}$ is valued in

$$
\bigcup_{i=1}^{I_{n}}\left[\mu_{-i}-C \delta^{2} \exp (-\gamma / \delta) ; \mu_{-i}+C \delta^{2} \exp (-\gamma / \delta)\right],
$$

for some $I_{n}<+\infty$. Therefore, to prove the result of this theorem, it is sufficient to show that for $\delta$ small enough, $\delta \mapsto \delta^{2} \lambda_{-n}^{\delta}$ does not meet the interval $\left[\mu_{-i}-C \delta^{2} \exp (-\gamma / \delta) ; \mu_{-i}+C \delta^{2} \exp (-\gamma / \delta)\right]$ for $i \neq n$. Let us proceed by contradiction assuming that there exists a subsequence $\left(\delta_{k}\right)$ such that $\lim _{k \rightarrow+\infty} \delta_{k}=0$ and $\lim _{k \rightarrow+\infty} \delta_{k}^{2} \lambda_{-n}^{\delta_{k}}=\mu_{\star} \in \mathfrak{S}\left(\mathrm{B}^{\infty}\right) \backslash \overline{\mathbb{R}_{+}}$, with $\mu_{\star} \neq \mu_{-n}$. Choosing $n$ closer to 1 if necessary, one may assume that there holds $\lim _{\delta \rightarrow 0} \delta^{2} \lambda_{-p}^{\delta}=\mu_{-p}$ for all $p=1,2, \ldots, n-1$ when $n \geq 2$. In other words, we consider the smallest $n \in \mathbb{N}^{*}$ such that the set $\left\{\delta^{2} \lambda_{-n}^{\delta}\right\}_{\delta \in\left(0 ; \delta_{0}\right]}$ has at least two distinct points of accumulation.

Notation. In the remainder of this proof, we will always consider values of $\delta \in\left\{\delta_{k}\right\}_{k \geq 0}$. To simplify notations, we will drop the subscript " $k$ " in " $\delta_{k}$ ". This should not bring confusion. On the other hand, in the sequel, the sequence $\left(w_{j}^{\delta}\right)_{j \in \mathbb{Z}}$ will refer to an orthonormal Hilbert basis of $\mathrm{L}^{2}\left(\Xi^{\delta}\right)$, where $w_{j}^{\delta}$ is an eigenfunction of $\mathrm{B}^{\delta}$ corresponding to $\delta^{2} \lambda_{j}^{\delta}$.

Step 1. Assume first that $\mu_{\star}>\mu_{-n}$. Let $\mathscr{E}^{\infty}$ refer to the eigenspace of $\mathrm{B}^{\infty}$ associated with the eigenvalue $\mu_{\star}$. This is a finite dimensional space because $\mu_{\star}$ does not belong to the essential spectrum of $\mathrm{B}^{\infty}$. We introduce $\mathbb{P}^{\infty}: \mathrm{L}^{2}\left(\mathbb{R}^{3}\right) \rightarrow \mathrm{L}^{2}\left(\mathbb{R}^{3}\right)$ the continuous linear map such that $\mathrm{Id}-\mathbb{P}^{\infty}$ is the spectral projector of $\mathrm{B}^{\infty}$ onto $\mathscr{E}^{\infty}$. On the other hand, we denote $J:=\left\{j \in \mathbb{N}^{*} \mid \mu_{-j}=\mu_{\star}\right\} \cup\{n\}$ and we set $\mathscr{F}^{\delta}:=\operatorname{span}\left\{\psi^{\delta} w_{j}^{\delta} \mid j \in J\right\} \subset D\left(\mathrm{~B}^{\infty}\right)$. Here and in the sequel, $\psi^{\delta}$ and $\chi^{\delta}=1-\psi^{\delta}$ are the cut-off functions defined in the proof of Lemma 5.4. Since $\mu_{\star}>\mu_{-n}$, we know that for all $\delta \in\left(0 ; \delta_{0}\right]$, there holds $\operatorname{card}(J)=\operatorname{dim} \mathscr{E}^{\infty}+1$, and that $\delta^{2} \lambda_{-j}^{\delta} \rightarrow \mu_{\star}$ for all $j \in J$ (here, we use the assumption that $n \in \mathbb{N}^{*}$ is the smallest index such that $\left\{\delta^{2} \lambda_{-n}^{\delta}\right\}_{\delta \in\left(0 ; \delta_{0}\right]}$ has at least two distinct points of accumulation). Moreover, remembering that $\left(w_{-j}^{\delta}\right)_{j \in \mathbb{Z}}$ is an orthonormal family and observing that $\lim _{\delta \rightarrow 0}\left\|\chi^{\delta} w_{-j}^{\delta}\right\|_{\mathbb{R}^{3}}=0$ (use the same arguments as in the proof of Lemma 5.4), one can show that $\left(\psi^{\delta} w_{-j}^{\delta}\right)_{j \in J}$ is linearly independent for $\delta$ small enough. This implies $\operatorname{dim} \mathscr{F}^{\delta}>\operatorname{dim} \mathscr{E}^{\infty}$. As a consequence, according to [15, Thm 1.1], there exists $v^{\delta} \in \mathscr{F}^{\delta}$ such that $\left\|v^{\delta}\right\|_{\mathbb{R}^{3}}=1$ and $\operatorname{dist}\left(v^{\delta}, \mathscr{E} \infty\right):=\inf \left\{\left\|v^{\delta}-w\right\|_{\mathbb{R}^{3}} \mid w \in \mathscr{E} \infty\right\}=1$, which can be rewritten $\left\|\mathbb{P}^{\infty} v^{\delta}\right\|_{\mathbb{R}^{3}}=1$, for $\delta>0$ small enough. Using again that $\lim _{\delta \rightarrow 0}\left\|\chi^{\delta} w_{-j}^{\delta}\right\|_{\mathbb{R}^{3}}=0$, we see that in the decomposition

$$
v^{\delta}=\sum_{j \in J} \alpha_{j}^{\delta} \psi^{\delta} w_{-j}^{\delta},
$$

where $\alpha_{j}^{\delta} \in \mathbb{C}$, there holds $\sum_{j \in J}\left|\alpha_{j}^{\delta}\right|^{2} \rightarrow 1$ when $\delta \rightarrow 0$. Denoting $C=\left(\operatorname{dist}\left(\mu_{\star}, \mathfrak{S}\left(\mathrm{B}^{\infty}\right) \backslash\left\{\mu_{\star}\right\}\right)\right)^{-2}$, we can write

$$
\begin{aligned}
1=\left\|\mathbb{P}^{\infty} v^{\delta}\right\|_{\mathbb{R}^{3}}^{2} & \leq C\left\|\left(\mathrm{~B}^{\infty}-\mu_{\star} \mathrm{Id}\right) \mathbb{P}^{\infty} v^{\delta}\right\|_{\mathbb{R}^{3}}^{2} \\
& \leq C\left\|\left(\mathrm{~B}^{\infty}-\mu_{\star} \mathrm{Id}\right) v^{\delta}\right\|_{\mathbb{R}^{3}}^{2} \\
& \leq C^{\prime} \sum_{j \in J}\left|\alpha_{j}^{\delta}\right|^{2}\left\|\left(\mathrm{~B}^{\infty}-\mu_{\star} \mathrm{Id}\right) \psi^{\delta} w_{-j}^{\delta}\right\|_{\mathbb{R}^{3}}^{2} \\
& \leq C^{\prime} \sum_{j \in J}\left|\alpha_{j}^{\delta}\right|^{2}\left(\left\|\left(\mathrm{~B}^{\infty}-\delta^{2} \lambda_{-j}^{\delta} \mathrm{Id}\right) \psi^{\delta} w_{-j}^{\delta}\right\|_{\mathbb{R}^{3}}^{2}+\left|\mu_{\star}-\delta^{2} \lambda_{-j}^{\delta}\right|^{2}\left\|\psi^{\delta} w_{-j}^{\delta}\right\|_{\mathbb{R}^{3}}^{2}\right) .
\end{aligned}
$$

We know, by assumption, that $\lim _{\delta \rightarrow 0}\left|\mu_{\star}-\delta^{2} \lambda_{-j}^{\delta}\right|=0$ for each $j \in J$. Moreover, we have shown in the proof of Lemma 5.4 that $\lim _{\delta \rightarrow 0}\left\|\left(\mathrm{~B}^{\infty}-\delta^{2} \lambda_{-j}^{\delta} \mathrm{Id}\right) \psi^{\delta} w_{-j}^{\delta}\right\|_{\mathbb{R}^{3}}=0$. As a consequence, the inequality written above leads to a contradiction, which concludes the first step of the proof.

Step 2. The only remaining possibility is that $\mu_{\star}<\mu_{-n}$. But recall that we have $\delta^{2} \lambda_{-j}^{\delta} \leq \delta^{2} \lambda_{-n}^{\delta}$ for all $j \geq n$. On the other hand, there holds $\lim _{\delta \rightarrow 0} \delta^{2} \lambda_{-p}^{\delta}=\mu_{-p}$ for all $p=1,2, \ldots, n-1$ (at least, when $n \geq 2)$. Therefore, $\mu_{\star}<\mu_{-n}$ implies that for $\delta_{0}$ small enough, we have $\left\{\delta^{2} \lambda_{-n}^{\delta}\right\}_{\delta \in\left(0 ; \delta_{0}\right]} \cap\left[\mu_{-n}-\right.$ $\left.\delta ; \mu_{-n}+\delta\right]=\emptyset$. This is in contradiction with Proposition 5.1. We conclude that the case $\mu_{\star}<\mu_{-n}$ is not possible either, which finishes the proof. 


\section{Numerical experiments in 2D}

In this section, we approximate numerically the spectrum of Problem (1) set in a $2 \mathrm{D}$ domain. We consider a $2 \mathrm{D}$ configuration for computational reasons, the number of degrees of freedom being too large in 3D. Although the analysis is slightly more involved, we can prove that all the results we have established in 3D for the spectrum of $\mathrm{A}^{\delta}$ also hold in 2D. We shall keep the same notations. First, we detail the parameters used for the numerical ex-

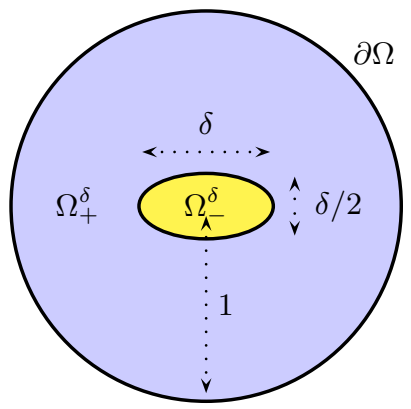

Figure 3: Geometry of the domains. periments. Let $\Omega \subset \mathbb{R}^{2}$ be the disk of radius one centered at $O$. Define the ellipse $\Xi_{-}:=\{(x, y) \in$ $\left.\mathbb{R}^{2} \mid(x / a)^{2}+(y / b)^{2}<1\right\}$ with $a=1 / 2$ and $b=1 / 4$. For $\delta \in(0 ; 1]$, set $\Omega_{-}^{\delta}:=\delta \Xi_{-}$and $\Omega_{+}^{\delta}:=\Omega \backslash \bar{\Omega}_{-}^{\delta}$ (see Figure 3). Introduce the function $\sigma^{\delta}: \Omega \rightarrow \mathbb{R}$ such that $\sigma^{\delta}=\sigma_{ \pm}$in $\Omega_{ \pm}^{\delta}$, where $\sigma_{+}=1$ and where $\sigma_{-}<0$ is a constant. We are interested in the $2 \mathrm{D}$ version of the eigenvalue problem (1) whose variational formulation writes

$$
\mid \begin{aligned}
& \text { Find }\left(\lambda^{\delta}, u^{\delta}\right) \in \mathbb{C} \times\left(\mathrm{H}_{0}^{1}(\Omega) \backslash\{0\}\right) \text { such that } \\
& \left(\sigma^{\delta} \nabla u^{\delta}, \nabla v\right)_{\Omega}=\lambda^{\delta}\left(u^{\delta}, v\right)_{\Omega}, \quad \forall v \in \mathrm{H}_{0}^{1}(\Omega) .
\end{aligned}
$$

Let us explain how to choose $\sigma_{-}$so that Assumption 1 holds. Using [21, Prop 8], when the contrast $\kappa_{\sigma}=\sigma_{-} / \sigma_{+}<0$ verifies $\kappa_{\sigma} \neq-1$, we can prove that the near field operator $\mathrm{B}^{\infty}$ defined in (10) is injective if and only if ${ }^{2}$

$$
\kappa_{\sigma} \notin \mathscr{S}:=\left\{\eta_{k}\right\}_{k=1}^{+\infty} \cup\left\{1 / \eta_{k}\right\}_{k=1}^{+\infty}, \quad \text { where } \quad \eta_{k}:=\frac{|a-b|^{k}+|a+b|^{k}}{|a-b|^{k}-|a+b|^{k}} .
$$

It is straightforward to check that $\left(\eta_{k}\right)_{k}$ is a sequence of numbers smaller than -1 , strictly increasing, which accumulates in -1 . Moreover, for the selected geometry, we have $\eta_{1}=-a / b=-2$. In order to satisfy Assumption 1, we will impose $\sigma_{-}=-2.5$ so that $\kappa_{\sigma}$ avoids the critical set $\mathscr{S}$. Now, we discretize Problem (51). Let us consider $\Omega_{h}, \Omega_{ \pm, h}^{\delta}$ polygonal approximations of the domains $\Omega, \Omega_{ \pm}^{\delta}$ satisfying $\overline{\Omega_{h}}=\overline{\Omega_{+, h}^{\delta}} \cup \overline{\Omega_{-, h}^{\delta}}$. We denote $\sigma_{h}^{\delta}: \Omega_{h} \rightarrow \mathbb{R}$ the function such that $\sigma_{h}^{\delta}=\sigma_{ \pm}$in $\Omega_{ \pm, h}^{\delta}$. Introduce $\left(\mathcal{T}_{h}^{\delta}\right)_{h}$ a shape regular family of triangulations of $\overline{\Omega_{h}}$, made of triangles. Here, $h$ refer to the mesh size. Moreover, we assume that, for any triangle $\tau$, one has either $\tau \subset \overline{\Omega_{+, h}^{\delta}}$ or $\tau \subset \overline{\Omega_{-, h}^{\delta}}$. Define the family of finite element spaces

$$
\mathrm{V}_{h}^{\delta}:=\left\{v \in \mathrm{H}_{0}^{1}\left(\Omega_{h}^{\delta}\right) \text { such that }\left.v\right|_{\tau} \in \mathbb{P}_{1}(\tau) \text { for all } \tau \in \mathcal{T}_{h}^{\delta}\right\},
$$

where $\mathbb{P}_{1}(\tau)$ is the space of polynomials of degree at most 1 on the triangle $\tau$. Let us consider the problem

$$
\mid \begin{aligned}
& \text { Find }\left(\lambda_{h}^{\delta}, u_{h}^{\delta}\right) \in \mathbb{C} \times\left(\mathrm{V}_{h}^{\delta} \backslash\{0\}\right) \text { such that } \\
& \left(\sigma_{h}^{\delta} \nabla u_{h}^{\delta}, \nabla v_{h}^{\delta}\right)_{\Omega_{h}^{\delta}}=\lambda_{h}^{\delta}\left(u_{h}^{\delta}, v_{h}^{\delta}\right)_{\Omega_{h}^{\delta}}, \quad \forall v_{h}^{\delta} \in \mathrm{V}_{h}^{\delta} .
\end{aligned}
$$

For details concerning the discretization process, we refer the reader to [6, 37, 12]. Nevertheless, we should emphasize that in these three papers, only the source term problem associated with (51) is considered. To this day, it seems that there exists no proof of convergence (as the mesh size $h$ tends to zero) of the standard $\mathbb{P}_{1}$ Lagrange finite element method to approximate the spectral Problem (51). It stems from the fact that the sign-changing of $\sigma^{\delta}$ prevents the use of usual approaches. However, from a practical point of view, for a given $\delta$, we observe no particular phenomena due to the presence of negative material and the eigenvalues look correctly approximated as $h \rightarrow 0$ (see Table 1). For

\footnotetext{
${ }^{2}$ Notice that when $\Xi_{-}$is a disk, i.e. when $a=b$, the set $\mathscr{S}$ reduces to $\{-1\}$.
} 
the computations, we use the FreeFem $++^{3}$ software while we display the results with Matlab $b^{4}$ and Paraview ${ }^{5}$.

\begin{tabular}{|c|c|c|}
\hline mesh size $h$ & $\lambda_{h,+1}^{\delta}$ & $\lambda_{h,-1}^{\delta}$ \\
\hline 0.0856833 & 5.90764 & -3.33104 \\
\hline 0.041797 & 5.85259 & -4.47873 \\
\hline 0.0214048 & 5.8386 & -4.80557 \\
\hline 0.00932046 & 5.83554 & -4.87614 \\
\hline 0.0061639 & 5.83511 & -4.88572 \\
\hline
\end{tabular}

Table 1: First positive and negative eigenvalues for several meshes with $\delta=0.5$. This suggests a convergence of eigenvalues in $O\left(h^{2}\right)$ toward their limit, which is in good agreement with the rate of convergence predicted by the Babuska-Osborn theory [2] for the case of $\mathbb{P}_{1}$ discretization of eigenvalue problems associated to compact self-adjoint operators.

In the next numerical experiments, we shall fix the mesh size $h$ so that the number of triangles inside the small inclusion $\Omega_{-, h}^{\delta}$ remains approximately constant. As a consequence, when $\delta$ goes to zero, the mesh contains more and more triangles. We make this choice so as to capture the localized eigenfunctions associated with the negative eigenvalues (see Figure 7).

In Figure 4, we display the positive eigenvalues of smallest modulus of Problem (52) with respect to $-\log _{10} \delta$. The dotted lines represent the approximation of the eigenvalues of smallest modulus of the limit operator $\mathrm{A}^{0}$ defined in (6). In other words, these dotted lines correspond to the spectrum of the problem without the inclusion of negative material. We observe that $\mathfrak{S}\left(\mathrm{A}^{\delta}\right)$ seems to converge to $\mathfrak{S}\left(\mathrm{A}^{0}\right)$ when the inclusion shrinks. This is in accordance with the analog of Theorem 4.1 in 2D.

In Figure 5, we present the behaviour of the negative eigenvalues of smallest modulus of Problem (52) with respect to $-\log _{10} \delta$. The numerical experiment suggests that the negative eigenvalues of the operator $\mathrm{A}^{\delta}$ tend to $-\infty$ when $\delta$ goes to zero. This was established in 3D in Lemma 5.1. Figure 6 confirms and clarifies this result: it indicates that the negative eigenvalues of Problem (52) behave like $\delta^{-2} \mu$, for some constant $\mu<0$, when $\delta \rightarrow 0$. This is coherent with the $2 \mathrm{D}$ version of Theorem 5.1.

Eventually, on Figure 7, we display the eigenfunctions associated with the negative and positive eigenvalues of smallest modulus for $\delta=0.5$ and for $\delta=0.05$. The eigenfunction associated with the smallest positive eigenvalue appears independent of the size of the small inclusion. On the contrary, we can observe the localization effect for the eigenfunction associated with the larger negative eigenvalue: when $\delta$ tends to zero, this eigenfunction is more and more concentrated around the inclusion of negative material.

\footnotetext{
${ }^{3}$ FreeFem++, http://www.freefem.org/ff++/.

${ }^{4}$ Matlab, http://www. mathworks.se/.

${ }^{5}$ Paraview, http://www . paraview.org/.
} 


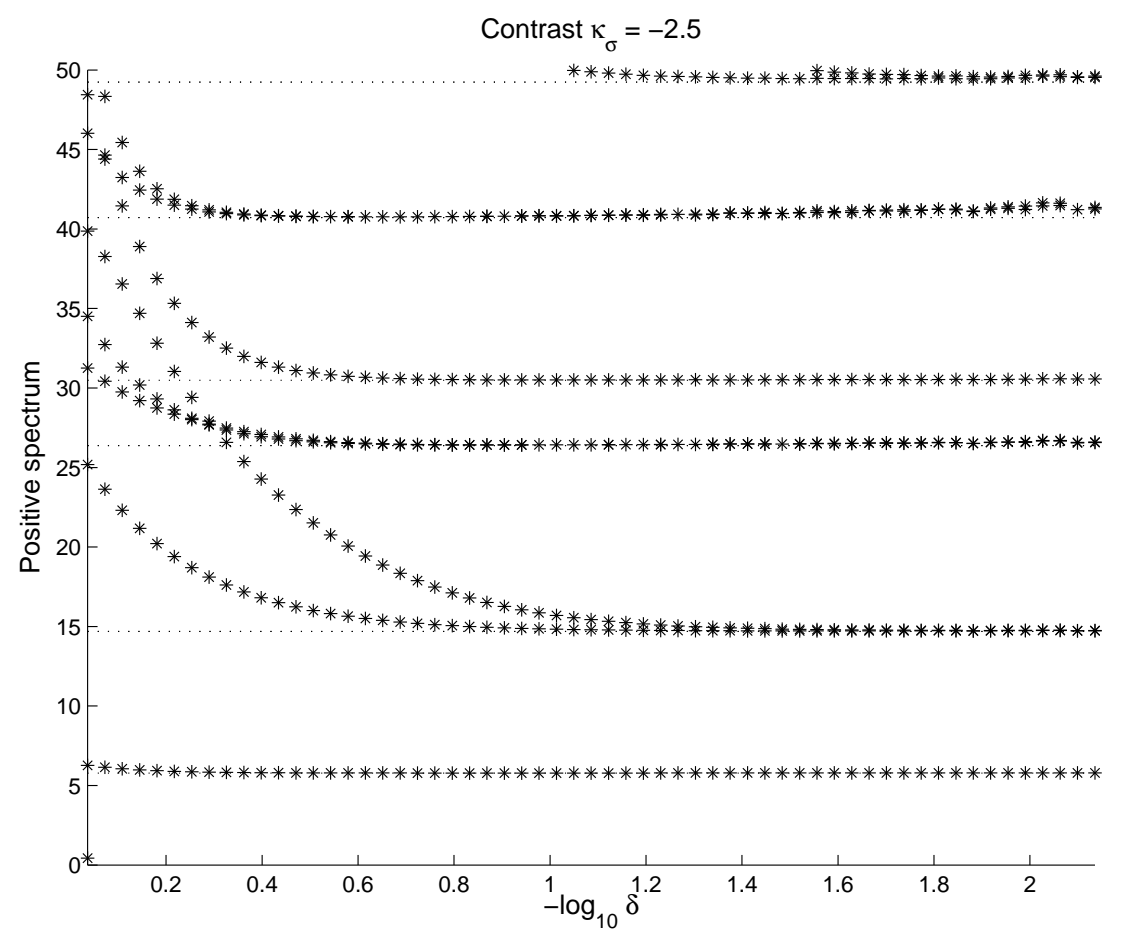

Figure 4: For a given $\delta \in(0 ; 1]$, we approximate the eigenvalues of smallest modulus of the operator $\mathrm{A}^{\delta}$. Then, we make $\delta$ tend to zero. The figure represents the approximation of the positive spectrum of $\mathrm{A}^{\delta}$ with respect to $-\log _{10} \delta$. The dotted lines correspond to the approximation of the eigenvalues of smallest modulus of the limit operator $\mathrm{A}^{0}$.

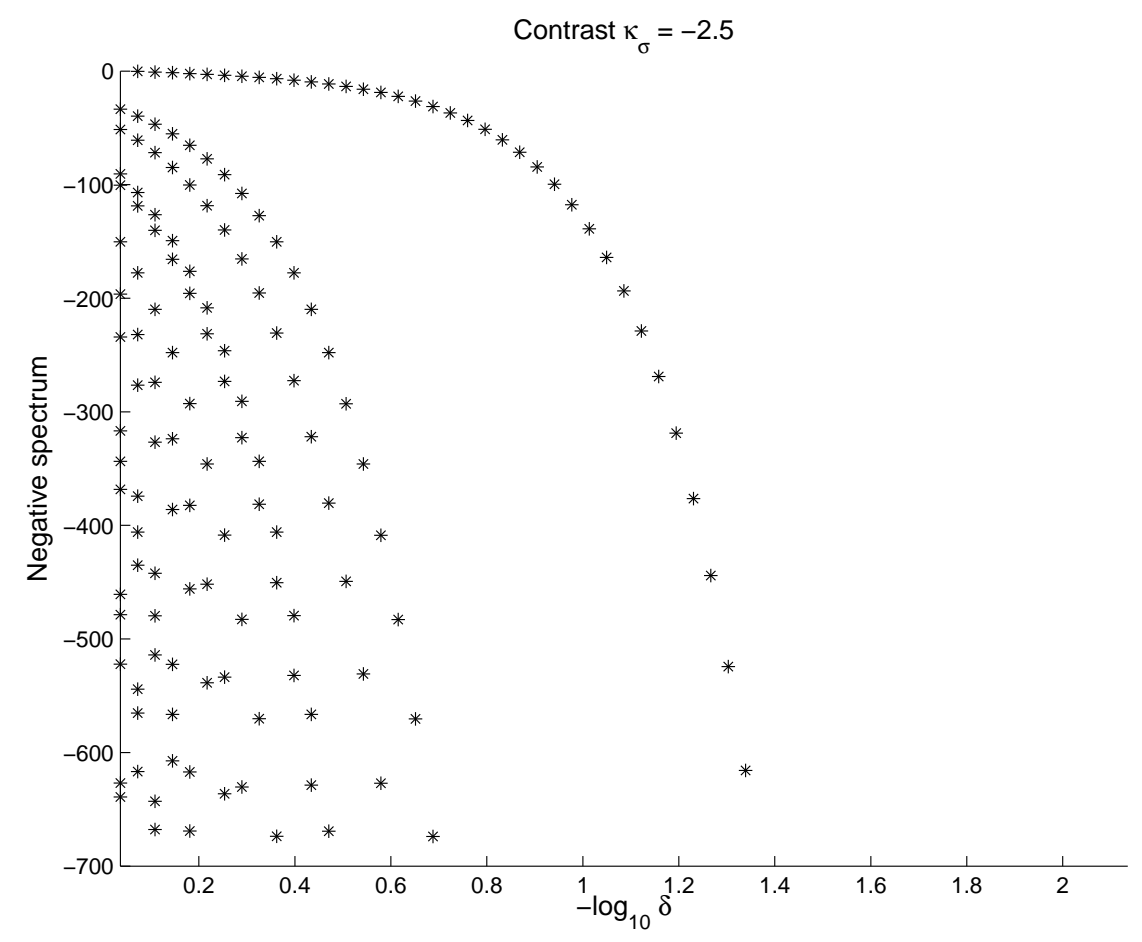

Figure 5: For a given $\delta \in(0,1]$, we approximate the eigenvalues of smallest modulus of the operator $\mathrm{A}^{\delta}$. Then, we make $\delta$ tend to zero. The figure represents the approximation of the negative spectrum of $\mathrm{A}^{\delta}$ with respect to $-\log _{10} \delta$. 


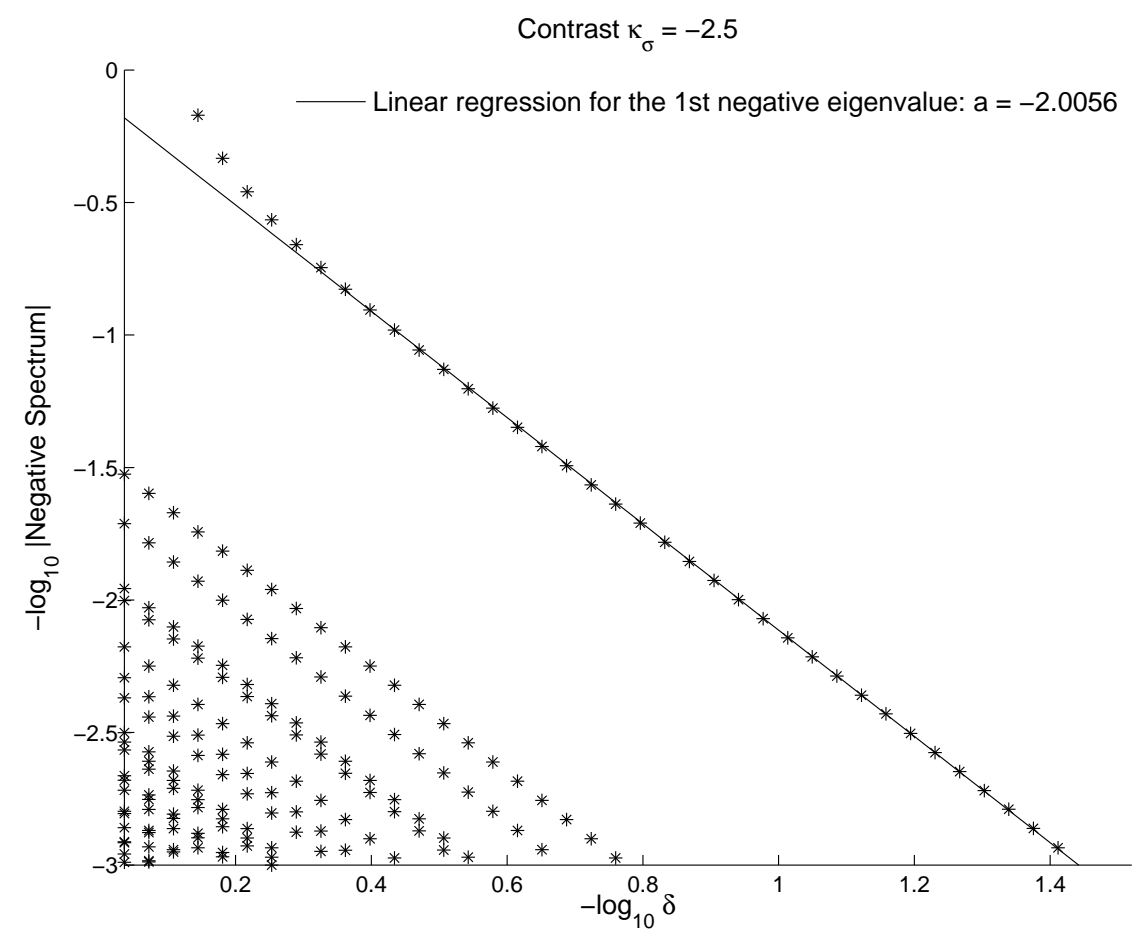

Figure 6: The figure represents the approximation of the negative eigenvalues of smallest modulus of $\mathrm{A}^{\delta}$ in logarithmic scale.
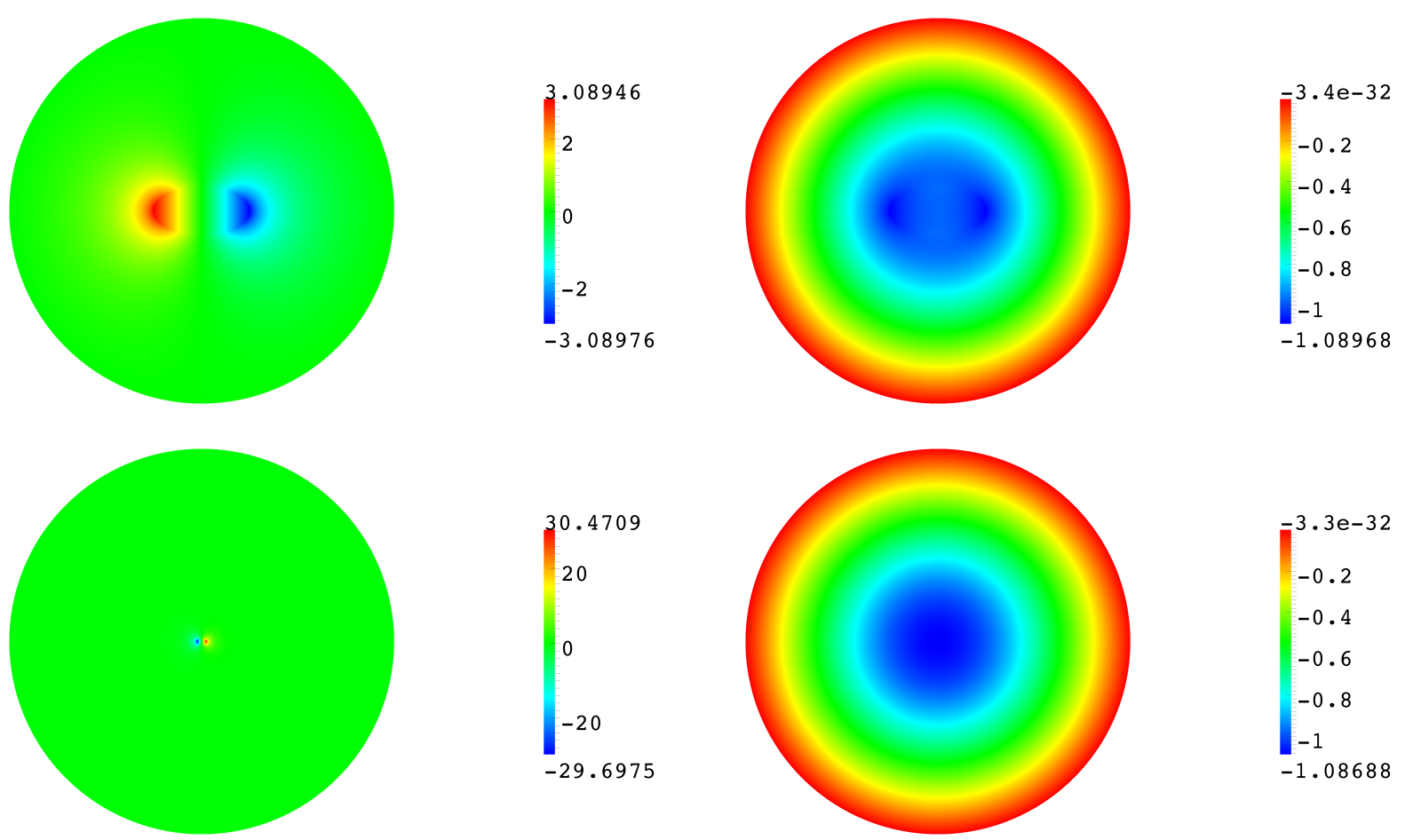

Figure 7: On the left (resp. on the right), approximation of the eigenfunction associated with the negative (resp. positive) eigenvalue of smallest modulus. Above, $\delta=0.5$. Below, $\delta=0.05$. The contrast $\kappa_{\sigma}=\sigma_{-} / \sigma_{+}$is chosen equal to -2.5 . We observe clearly the localization effect for the eigenfunctions associated with the negative eigenvalues.

\section{Appendix}

In this appendix, we briefly recall an elementary result of spectral theory that we used three times in this article in order to estimate the distance of a number to the spectrum of an operator. We provide 
a proof for the sake of completeness.

Lemma 6.1. Let $\mathrm{H}$, equipped with the inner product $(\cdot, \cdot)_{\mathrm{H}}$ and the norm $\|\cdot\|_{\mathrm{H}}$, be a Hilbert space. For any (a priori unbounded) normal linear operator $\mathrm{A}: D(\mathrm{~A}) \subset \mathrm{H} \rightarrow \mathrm{H}$ we have

$$
\inf _{\lambda \in \mathfrak{S}(\mathrm{A})}|\lambda-\mu| \leq \inf _{v \in D(\mathrm{~A}) \backslash\{0\}} \frac{\|\mathrm{A} v-\mu v\|_{\mathrm{H}}}{\|v\|_{\mathrm{H}}}, \quad \forall \mu \in \mathbb{C} .
$$

Proof. Since A is normal then, according to the spectral theorem [4, Thm 6.6.1], it admits a spectral decomposition $\mathrm{A}=\int_{\mathfrak{S}(\mathrm{A})} \zeta d \mathrm{E}(\zeta)$ where $\mathrm{E}(\zeta)$ refers to a spectral measure on $\mathrm{H}$. Let $d \mathrm{E}_{v, v}$ refer to the measure associated with $\zeta \mapsto(\mathrm{E}(\zeta) v, v)_{\mathrm{H}}$. A spectral decomposition of A $-\mu \mathrm{Id}$ is given by $\mathrm{A}-\mu \mathrm{Id}=\int_{\mathfrak{S}(\mathrm{A})}(\zeta-\mu) d \mathrm{E}(\zeta)$. Moreover the formula $\|\mathrm{A} v-\mu v\|_{\mathrm{H}}^{2}=\int_{\mathfrak{S}(\mathrm{A})}|\zeta-\mu|^{2} d \mathrm{E}(\zeta)$ holds for any $v \in D(\mathrm{~A})$. As a consequence, we have

$$
\|v\|_{\mathrm{H}}^{2} \inf _{\lambda \in \mathfrak{S}(\mathrm{A})}|\lambda-\mu|^{2}=\inf _{\lambda \in \mathfrak{S}(\mathrm{A})}|\lambda-\mu|^{2} \int_{\mathfrak{S}(\mathrm{A})} d \mathrm{E}_{v, v}(\zeta) \leq \int_{\mathfrak{S}(\mathrm{A})}|\zeta-\mu|^{2} d \mathrm{E}_{v, v}(\zeta)=\|\mathrm{A} v-\mu v\|_{\mathrm{H}}^{2}
$$

Since this holds for any $v \in D(\mathrm{~A})$, we can divide by $\|v\|_{\mathrm{H}}^{2}$ and take the inf in the right hand side of the estimate above, which yields the desired inequality.

\section{Acknowledgments}

The research of the two first authors is supported by the ANR project METAMATH, grant ANR11-MONU-016 of the French Agence Nationale de la Recherche. The work of the first author is also partially supported by the Academy of Finland (decision 140998) and by the FMJH through the grant ANR-10-CAMP-0151-02 in the "Programme des Investissements d'Avenir". The research of the third author is supported by the Russian Foundation for Basic Research, grant No. 12-01-00348.

\section{References}

[1] S. Anantha Ramakrishna. Physics of negative refractive index materials. Rep. Prog. Phys., 68(2):449-521, 2005.

[2] I. Babuska and J. Osborn. Eigenvalue problems. In Finite Element Methods (Part 1), volume 2 of Handbook of Numerical Analysis, pages 641 - 787. Elsevier, 1991.

[3] W.L. Barnes, A. Dereux, and T.W. Ebbesen. Surface plasmon subwavelength optics. Nature, 424(6950):824-830, 2003.

[4] M.Sh. Birman and M.Z. Solomjak. Spectral theory of selfadjoint operators in Hilbert space. Mathematics and its Applications (Soviet Series). D. Reidel Publishing Co., Dordrecht, 1987.

[5] A.-S. Bonnet-Ben Dhia, L. Chesnel, and P. Ciarlet Jr. T-coercivity for scalar interface problems between dielectrics and metamaterials. Math. Mod. Num. Anal., 46:1363-1387, 2012.

[6] A.-S. Bonnet-Ben Dhia, P. Ciarlet Jr., and C.M. Zwölf. Time harmonic wave diffraction problems in materials with sign-shifting coefficients. J. Comput. Appl. Math, 234:1912-1919, 2010. Corrigendum J. Comput. Appl. Math., 234:2616, 2010.

[7] A.-S. Bonnet-Ben Dhia and K. Ramdani. A non elliptic spectral problem related to the analysis of superconducting micro-strip lines. Math. Mod. Num. Anal., 36(3):461-487, 2002.

[8] E. Bonnetier and F. Triki. On the spectrum of the Poincaré variational problem for two close-totouching inclusions in 2D. Arch. Rational Mech. Anal., pages 1-27, 2013.

[9] J. Cainzos, E. Pérez, and M. Vilasánchez. Asymptotics for the eigenelements of the Neumann spectral problem with concentrated masses. Indiana Univ. Math. J., 56(4):1939-1987, 2007.

[10] G. Cardone, T. Durante, and S.A. Nazarov. The localization effect for eigenfunctions of the mixed boundary value problem in a thin cylinder with distorted ends. SIAM J. Math. Anal., 42(6):2581-2609, 2010.

[11] R. Charbonneau, N. Lahoud, G. Mattiussi, and P. Berini. Demonstration of integrated optics elements based on long-ranging surface plasmon polaritons. Opt. Express, 13(3):977-984, 2005. 
[12] L. Chesnel and P. Ciarlet Jr. T-coercivity and continuous Galerkin methods: application to transmission problems with sign changing coefficients. Numer. Math., 124(1):1-29, 2013.

[13] L. Chesnel, X. Claeys, and S.A. Nazarov. A curious instability phenomenon for a rounded corner in presence of a negative material. Asymp. Anal., 88(1):43-74, 2014.

[14] M. Costabel and E. Stephan. A direct boundary integral method for transmission problems. $J$. of Math. Anal. and Appl., 106:367-413, 1985.

[15] I.C. Gohberg and M.G. Krein. The basic propositions on defect numbers, root numbers and indices of linear operators. Amer. Math. Soc. Transl., Ser. 2, 13:185-264, 1960.

[16] D.K. Gramotnev and S.I. Bozhevolnyi. Plasmonics beyond the diffraction limit. Nature Photon., $4(2): 83-91,2010$.

[17] D. Grieser. The plasmonic eigenvalue problem. Rev. Math. Phys., 26(3):1450005, 26, 2014.

[18] J. Helsing and K.-M. Perfekt. On the polarizability and capacitance of the cube. Appl. Comput. Harmon. Anal., 34(3):445-468, 2013.

[19] I.V. Kamotskii and S.A. Nazarov. On eigenfunctions localized in a neighborhood of the lateral surface of a thin domain. J. Math. Sci., 101(2):2941-2974, 2000.

[20] T. Kato. Perturbation theory for linear operators. Classics in Mathematics. Springer-Verlag, Berlin, 1995. Reprint of the 1980 edition.

[21] D. Khavinson, M. Putinar, and H.S. Shapiro. Poincaré's variational problem in potential theory. Arch. Rational Mech. Anal., 185(1):143-184, 2007.

[22] V.A. Kondratiev. Boundary-value problems for elliptic equations in domains with conical or angular points. Trans. Moscow Math. Soc., 16:227-313, 1967.

[23] J.-L. Lions and E. Magenes. Problèmes aux limites non homogènes et applications. Dunod, 1968.

[24] M. Lobo and E. Pérez. Local problems for vibrating systems with concentrated masses: a review. C. R., Méc., Acad. Sci. Paris, 331(4):303-317, 2003.

[25] S. Maier. Plasmonics: Fundamentals and Applications. Springer, 2007.

[26] V.G. Maz'ya and S.A. Nazarov. Singularities of solutions of the Neumann problem at a conic point. Sibirsk. Mat. Zh., 30(3):52-63, 218, 1989.

[27] V.G. Maz'ya, S.A. Nazarov, and B.A. Plamenevskiur. Singularities of solutions of the Dirichlet problem in the exterior of a thin cone. Mat. Sb. (N.S.), 122(164)(4):435-457, 1983.

[28] V.G. Maz'ya, S.A. Nazarov, and B.A. Plamenevskil. Asymptotic expansions of eigenvalues of boundary value problems for the Laplace operator in domains with small openings. Izv. Akad. Nauk SSSR Ser. Mat., 48(2):347-371, 1984.

[29] V.G. Maz'ya, S.A. Nazarov, and B.A. Plamenevskiu. Asymptotic theory of elliptic boundary value problems in singularly perturbed domains, Vol. 1, 2. Birkhäuser, Basel, 2000. Translated from the original German 1991 edition.

[30] S.A. Nazarov. Interaction of concentrated masses in a harmonically oscillating spatial body with neumann boundary conditions. Math. Model. Numer. Anal., 27(6):777-799, 1993.

[31] S.A. Nazarov. Asymptotic conditions at a point, selfadjoint extensions of operators, and the method of matched asymptotic expansions. In Proceedings of the St. Petersburg Mathematical Society, Vol. V, volume 193 of Amer. Math. Soc., Transl. Ser. 2, pages 77-125, Providence, RI, 1999.

[32] S.A. Nazarov. Asymptotics of negative eigenvalues of the Dirichlet problem with the density changing sign. J. Math. Sci. (N. Y.), 163(2):151-175, 2009.

[33] S.A. Nazarov. Localization near the corner point of the first eigenfunction of the Dirichlet problem in a domain with thin edging. Sibirsk. Mat. Zh., 52(2):350-370, 2011.

[34] S.A. Nazarov, I.L. Pankratova, and A.L. Piatnitski. Homogenization of the spectral problem for periodic elliptic operators with sign-changing density function. Arch. Ration. Mech. Anal., 200(3):747-788, 2011.

[35] S.A. Nazarov and A.L. Piatnitski. Homogenization of the spectral Dirichlet problem for a system of differential equations with rapidly oscillating coefficients and changing sign density. J. Math. Sci. (N. Y.), 169(2):212-248, 2010.

[36] S.A. Nazarov and B.A. Plamenevskiǔ. Elliptic problems in domains with piecewise smooth boundaries, volume 13 of Expositions in Mathematics. De Gruyter, Berlin, Germany, 1994. 
[37] S. Nicaise and J. Venel. A posteriori error estimates for a finite element approximation of transmission problems with sign changing coefficients. J. Comput. Appl. Math., 235(14):4272-4282, 2011.

[38] O.A. Oleinik, J. Sanchez-Hubert, and G.A. Yosifian. On vibrations of a membrane with concentrated masses. Bull. Sci. Math. SÃlr. 2, 115:1-27, 1991.

[39] M. A. Ordal, L. L. Long, R. J. Bell, S. E. Bell, R. R. Bell, R. W. Alexander, and C. A. Ward. Optical properties of the metals al, co, cu, au, fe, pb, ni, pd, pt, ag, ti, and w in the infrared and far infrared. Appl. Opt., 22(7):1099-1119, Apr 1983.

[40] J. Peetre. Another approach to elliptic boundary problems. Commun. Pure Appl. Math., 14:711$731,1961$.

[41] K.-M. Perfekt and M. Putinar. Spectral bounds for the Neumann-Poincaré operator on planar domains with corners. J. Anal. Math., 124:39-57, 2014.

[42] H. Poincaré. La méthode de Neumann et le problème de Dirichlet. Acta Math., 20(1):59-142, 1897.

[43] K. Ramdani. Lignes supraconductrices: analyse mathématique et numérique. PhD thesis, Université Paris 6, France, 1999.

[44] Ya.A. Roĭtberg and Z.G. Šeftel'. General boundary-value problems for elliptic equations with discontinuous coefficients. Dokl. Akad. Nauk SSSR, 148:1034-1037, 1963.

[45] E. Sanchez-Palencia. Perturbation of eigenvalues in thermoelasticity and vibration of systems with concentrated masses. In Trends and applications of Pure Mathematics to Mechanics, pages 346-368. Springer, 1984.

[46] L. Tartar. Sur un lemme d'équivalence utilisé en analyse numérique. Calcolo, 24(2):129-140, 1987.

[47] M.I. Vishik and L.A. Lyusternik. Regular degeneration and boundary layer for linear differential equations with small parameter. Amer. Math. Soc. Transl.(2), 20:239-364, 1962.

[48] A.V. Zayats, I.I. Smolyaninov, and A.A. Maradudin. Nano-optics of surface plasmon polaritons. Physics reports, 408(3-4):131-314, 2005. 